\title{
Review
}

\author{
$\mathrm{Hao} \mathrm{Hu*}$ and Leif K. Oxenløwe
}

\section{Chip-based optical frequency combs for high-capacity optical communications}

https://doi.org/10.1515/nanoph-2020-0561

Received October 7, 2020; accepted December 16, 2020; published online February 3, 2021

\begin{abstract}
Current fibre optic communication systems owe their high-capacity abilities to the wavelength-division multiplexing (WDM) technique, which combines data channels running on different wavelengths, and most often requires many individual lasers. Optical frequency combs, with equally spaced coherent comb lines derived from a single source, have recently emerged as a potential substitute for parallel lasers in WDM systems. Benefits include the stable spacing and broadband phase coherence of the comb lines, enabling improved spectral efficiency of transmission systems, as well as potential energy savings in the WDM transmitters. In this paper, we discuss the requirements to a frequency comb for use in a high-capacity optical communication system in terms of optical linewidth, per comb line power and optical carrier-to-noise ratio, and look at the scaling of a comb source for ultra-high capacity systems. Then, we review the latest advances of various chip-based optical frequency comb generation schemes and their applications in optical communications, including mode-locked laser combs, spectral broadening of frequency combs, microresonator-based Kerr frequency combs and electro-optic frequency combs.
\end{abstract}

Keywords: electro-optic frequency comb; fibre-optic communication; frequency comb; Kerr frequency comb; micro-comb; mode-locked laser; spectral broadening; wavelength division multiplexing (WDM).

*Corresponding author: Hao Hu, DTU Fotonik, Technical University of Denmark, Kgs. Lyngby, Denmark, E-mail: huhao@fotonik.dtu.dk. https://orcid.org/0000-0002-8859-0986

Leif K. Oxenløwe, DTU Fotonik, Technical University of Denmark, Kgs. Lyngby, Denmark

\section{Introduction}

An optical frequency comb has an optical spectrum consisting of a series of discrete, equally spaced and phase-locked frequency lines. Frequency combs can be generated in several ways, and have many applications, and have therefore attracted a lot of attention. In 2005, half of the Nobel Prize in physics was awarded to John L. Hall and Theodor W. Hansch for their pioneering work on optical frequency combs for spectroscopy applications. Since then, optical frequency combs have been widely investigated for numerous diverse applications, such as spectroscopy, ranging, atomic clocks, searching for exoplanets, microwave photonics and optical communications [1-16].

Current optical communication systems are based on wavelength-division multiplexing (WDM), which relies on arrays of discrete wavelength laser sources [17]. An optical frequency comb with a frequency spacing on the order of $10-100 \mathrm{GHz}$ can be used to replace the laser lines from individual sources in a WDM communication system, and may potentially replace hundreds of individual lasers, with the aim of reducing the energy consumption and size of the transmitter $[2,5]$. In addition, the intrinsic relative frequency stability of the frequency comb and the phase coherence between the comb lines can enable unique advantages over the individual lasers, such as increased spectral efficiency by reducing guard-bands or even achieving below zero guard-band, joint digital signal processing by sharing phase information, and mitigation of nonlinear impairments in the fibre link [18-20]. However, modern optical communication systems pose strict requirements on a frequency comb in terms of frequency spacing, optical linewidth and power per comb line, which must be fulfilled before replacing the lasers.

In this paper, we will first review the key parameters for evaluating the quality of an optical frequency comb in a high-capacity WDM optical communication system. Then, we will review various schemes for chip-based frequency comb generation, including chip-based mode-locked lasers, nano-waveguide-based frequency 
combs spectral broadening, microresonator-based Kerr frequency combs and thin-film lithium niobate-based integrated electro-optic (EO) frequency combs. Finally, we will summarize all the frequency comb generation schemes mentioned in the paper and discuss the potentials and issues for implementing chip-based frequency combs in real high-capacity WDM optical communication systems.

\section{Frequency combs as communication sources}

Coherent optical communications rely on laser sources with narrow linewidth and high optical signal to noise ratio (OSNR). The prospect of replacing a laser array with a frequency comb source is attractive, because a single comb source can produce hundreds of frequency lines with characteristics such as narrow linewidths (reproducing that of the pump laser, which can be chosen to be very narrow linewidth), adequate OSNR, locked frequency spacing between the lines and a stable phase relation between them. The frequency comb can be used as communication sources not only in the transmitter but also in the receiver, as shown in Figure 1.

The line frequency stability allows for reduced guard bands in WDM transmission systems, thus increasing the spectral efficiency [5]. The additional line phase stability allows for multi-channel linear digital signal processing (DSP) of coherent communication signals, which has successfully been used to demonstrate increased spectral efficiency, and increased transmission reaches [19]. A coherent comb source has the key feature that all lines are stably equidistant and have the same initial phase, as opposed to a laser array, and this enables the reuse of
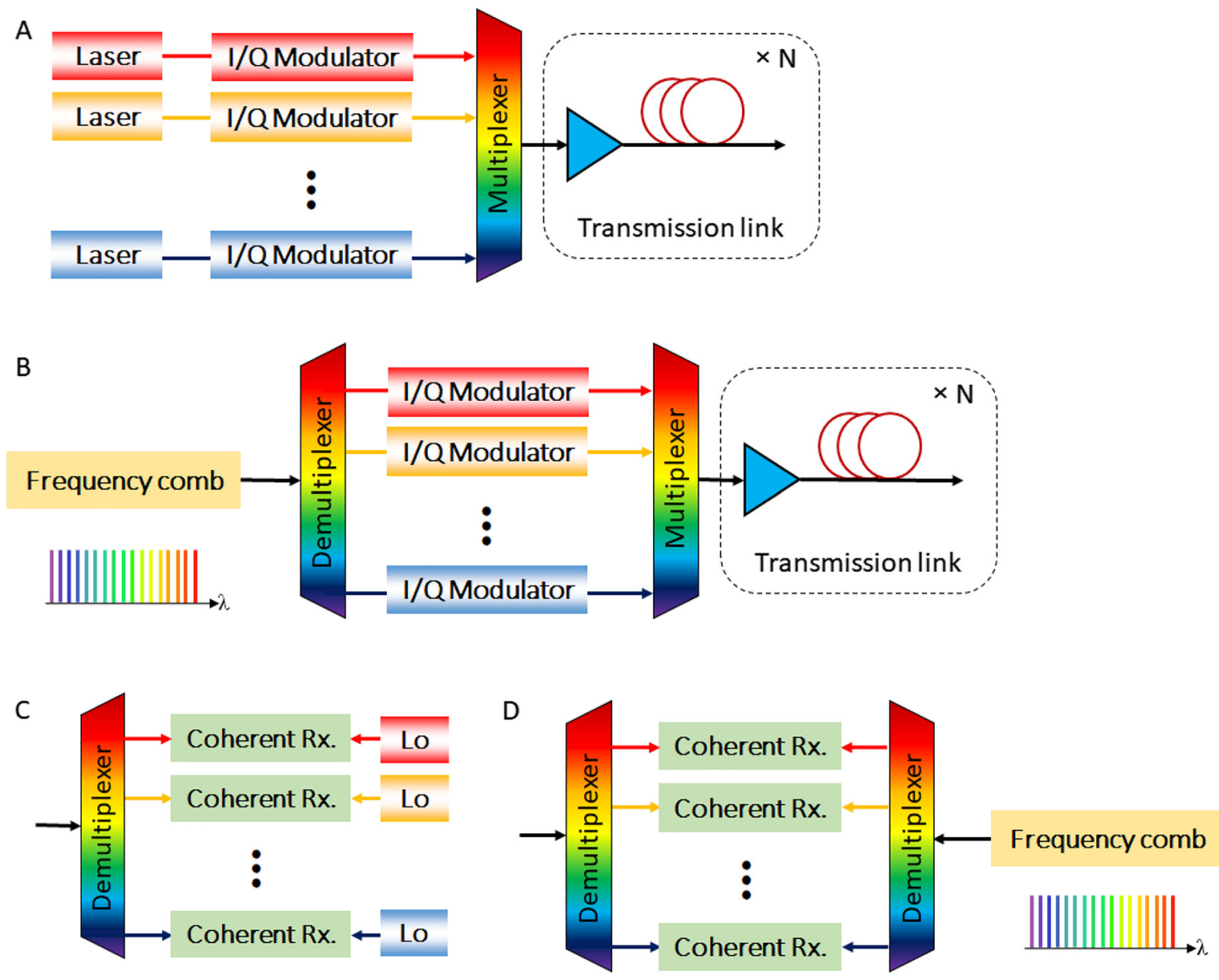

Figure 1: Schematic of high-speed WDM coherent optical communication systems.

(A) Conventional WDM transmitter using individual lasers at different wavelengths as sources. (B) A frequency comb source-based WDM transmitter. (C) Conventional WDM coherent receiver using individual lasers as local oscillators (LOs). (D) A frequency comb source-based WDM coherent receiver. 
phase information retrieved from one channel to other channels [5]. The frequency and phase stability may thus be utilized to directly mitigate interchannel interference, by using the information of the jointly detected sidechannels to mitigate their crosstalk on the central channel, and even enable a reduction of the guardbands below zero [19]. The joint DSP is also seen to reduce the required processing power per channel, and hence increase the overall energy efficiency of the communication system [5]. Additionally, clear signs of improvement of transmission performance have been observed and characterized in the nonlinear fibre transmission regime, partly relying on frequency locked comb lines in a digital pre-compensation scheme [20]. As discussed in the studies by Galdino et al. and Semrau et al. [21, 22], the transmitter noise and receiver noise plays an important part in digital back propagation, with a small advantage for pre-compensation, and this may even be emphasized more if the transmitter noise is reduced. This may be aided by e.g. using very clean laser frequency lines, depending on the overall noise profile through the transmitter.

\subsection{Optical linewidth}

Linewidths of $100 \mathrm{kHz}$ are easily attainable and perfectly adequate for 256 QAM data modulation [5], and even narrower linewidths allows for either higher QAM orders [23] or much simplified DSP, with the extreme case of foregoing linear phase noise correction altogether for linewidths in the sub-kHz range and for 256 QAM [23]. Compared to ECLs and DFB lasers, in the context of achievable information rate (AIR) and optical phase conjugation transmission systems, 1-2 dB of OSNR gain has been achieved for a sub$\mathrm{kHz}$ laser [24]. Again, using such narrow linewidth lasers as the seed in a comb generator provides a source of multiple narrow linewidth lines with these attractive features.

\subsection{Power per comb line and OSNR}

We investigate power per comb line for various length of transmission, as shown in Figure 2, and compare to the case of a single laser with enough power to not need additional amplifiers before launching into the transmission fibre with $0 \mathrm{dBm}$ optical power per line, inspired by [5]. A comb line with realistic powers of $-10 \mathrm{dBm}$ or less straight out of the comb source, and an optical carrier to noise ratio (OCNR estimated as power per comb line relative to the quantum noise limit of $-58 \mathrm{dBm}$ ) of about $45 \mathrm{~dB}$, needs a pre-amplifier as well as a compensating amplifier after the lossy data modulator before launch at $0 \mathrm{dBm}$ power into the transmission span, which reduces the OSNR. Note that a polariser in front of the modulator reduces the noise sent to the modulator. One could also consider using an optical filter to reduce the ASE noise further, but this would be practically challenging and thus omitted in this analysis. Assuming EDFA noise figures of $4.5 \mathrm{~dB}$, and transmission spans of $80 \mathrm{~km}$ with link EDFAs to compensate the loss in the fibre $(0.2 \mathrm{~dB} / \mathrm{km})$, Figure $2 \mathrm{~B}$ displays the evolution of the OSNR for the laser case and power per comb line cases of $-10,-20,-30$, and $-40 \mathrm{dBm}$. The laser, with fewer amplifiers in the transmission line, yields the highest possible OSNR. At short reach it's about $2 \mathrm{~dB}$ better than the $-10 \mathrm{dBm}$ OSNR per comb line. The $-20 \mathrm{dBm}$ OSNR per comb line is yet $2 \mathrm{~dB}$ lower than the $-10 \mathrm{dBm}$ case, but for both -10 and $-20 \mathrm{dBm}$ the gap to the laser OSNR quickly diminishes with more transmission links. After about 12 spans the -th $\mathrm{dBm}$ case has less than 1-dB penalty to the laser case, and after about 15 spans, the - ca $\mathrm{dBm}$ case has less than $1-\mathrm{dB}$ penalty to the laser case. So after about $1000 \mathrm{~km}$, the comb case is pretty much as good as the laser case. This is because the overall transmission gets more and more dominated by the many EDFAs in the link. Figure $2 \mathrm{C}$ shows a plot of the required power per comb line, $P_{0}$, to maintain a 1-dB OSNR penalty after transmission of the comb case to the case of using individual lasers. As seen, going beyond about 20 links, $1600 \mathrm{~km}$, combs with line powers less than $-25 \mathrm{dBm}$ suffice to create high-quality transmission. For trans-Atlantic distances, $-30 \mathrm{dBm}$ (OCNR of $28 \mathrm{~dB}$ ) or less is enough. Figure 2D shows the difference in $\mathrm{dB}$ of the comb OSNR to the laser OSNR as a function of power per comb line for varying numbers of links. Generally, at short reach there is a large penalty of several $\mathrm{dB}$, which grows with lowering the power per comb line. For longer reach and high power, this difference decreases. A plateau is seen, on which there is a low penalty to the laser case. This plateau shows there is a large region where a comb source could offer good value for money. It is worth noting that $-13 \mathrm{dBm}$ power per comb line is feasible and has been experimentally demonstrated [25], but as mentioned, even for $-20 \mathrm{dBm}$ after 15 spans the penalty to a laser is less than $1 \mathrm{~dB}$ and at short reach, $-10 \mathrm{dBm}$ is about $2 \mathrm{~dB}$ worse than the laser case. So even for short reach, or a single link, it is possible to get very decent OSNRs for a range of reasonable levels of powers per comb line (more than $30 \mathrm{~dB}$ OSNR).

So depending on the need, one can determine the requirements to the power per comb line this way. E.g. if $25 \mathrm{GBd}$ is used, $31 \mathrm{~dB}$ OSNR will be needed to support 256 QAM at a bit error rate below $1 \mathrm{E}-3$, yielding a generalized mutual information (GMI) of 8 bits/symbol. This can 


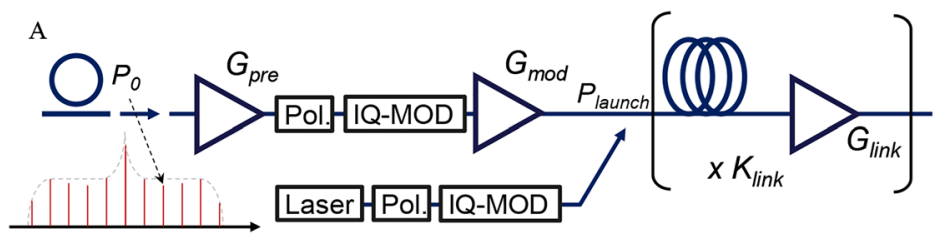

B
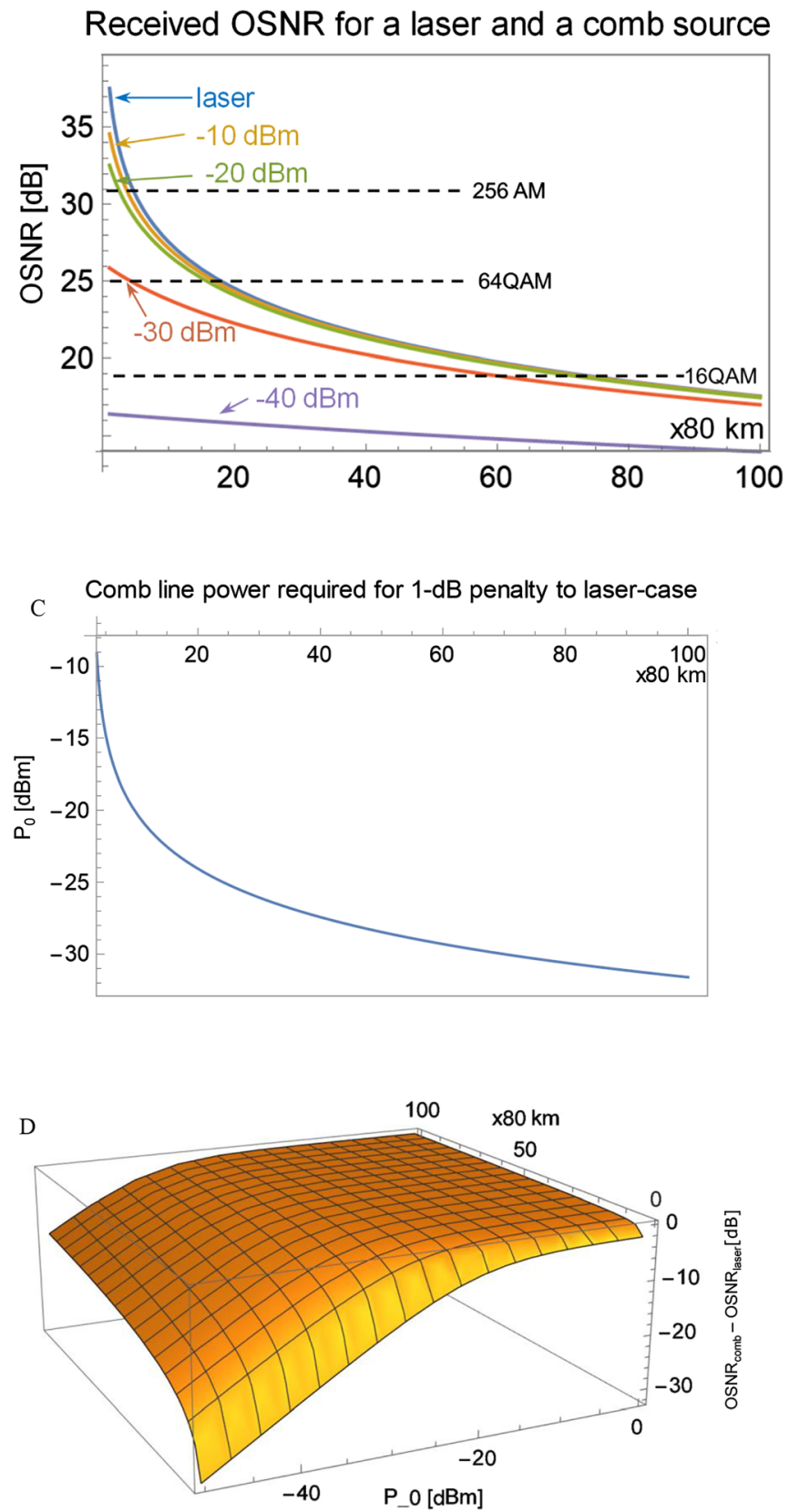

Figure 2: Investigating the power per comb line for various transmission reaches.

(A) Schematic setup of the investigated systems, comparing a comb source, e.g. a ring resonator, to a laser with high enough power to neither need a pre-amplifier nor an amplifier to compensate for the losses in the modulator. A polariser is assumed in front of the modulator, essentially rejecting half of the noise prior to that point. The launch power is set to $0 \mathrm{dBm}$. (B) Receiver OSNR for various powers per comb line compared to the laser case, and inserted dashed lines for OSNR levels required to support 256, 64, 16 QAM. For the 256 QAM case, the laser can go $4.6 \times 80 \mathrm{~km}$, the $-10-\mathrm{dBm}$ comb case can go $3.5 \times 80 \mathrm{~km}$ and the $-20-\mathrm{dBm}$ comb case can go $2.4 \times 80 \mathrm{~km}$ with high enough received OSNR to sustain the high order modulation. (C) The required power per comb line for a 1-dB OSNR penalty compared to the laser case. (D) Comparison of the comb OSNR to the laser OSNR for different powers per comb line as a function of transmission reach.

be supported by $-10 \mathrm{dBm}$ power per comb line. For 64 QAM, $-20 \mathrm{dBm}$ power per comb line is adequate. These powers per comb line are attainable by several existing comb sources. Note that, higher spectral efficiency can be achieved with a narrower frequency spacing and smaller symbol rate [26]. 


\subsection{Multiplexing capacity}

The calculated capacity shown in Figure 3B suggests a potential achievable capacity of $989 \mathrm{Tbit} / \mathrm{s}$ for this setup. The experimental conditions were not suitable to try to go closer to capacity than $661 \mathrm{Tbit} / \mathrm{s}$, so as expected there was an implementation penalty. The curve in Fig. B shows that for unrepeated transmission, one could still obtain about $800 \mathrm{Tbit} / \mathrm{s}$ over more than $50 \mathrm{~km}$.

As discussed, the achievable power per comb line sets a limit on how much data can be carried by each line, and the number of generated lines and their spacing (free spectral range) compared to the realistic data modulation rate, dictates the achievable WDM capacity. Since each line can be separated without loss of power, using wavelength splitters and filters, the scaling of the total capacity for WDM is simply the number of lines multiplied with the single line capacity.

For space division multiplexing with a single comb source, each line will be power divided into the number of desired spatial channels, and hence each channel will have equivalently less line power, and accordingly lower OSNR. However, the number of channels is increased, and so the overall capacity can still grow, until the line power is depleted.

The total capacity for SDM and WDM becomes:

$$
C_{\text {total }}=n_{\mathrm{WDM}} m_{\mathrm{SDM}} R_{\mathrm{S}} \log _{2}(1+\mathrm{SNR})
$$

where $n_{\mathrm{WDM}}$ is the total number of WDM lines, $\mathrm{m}_{\mathrm{SDM}}$ is the total number of SDM channels, $R_{s}$ is the modulation symbol rate, and the SNR is directly related to the OSNR shown in Figure 2, as the noise bandwidth $B_{\text {ref }}$ divided by the modulation rate $R_{s}$. For SDM, using a single comb source, the SNR will decrease with more SDM channels, due to the power splitting, so the SDM capacity increase depends on terms both inside and outside the logarithmic function, and it is expected that $C$ will saturate for a certain $P_{0}$ and a given number of SDM channels, $m_{\mathrm{SDM}}$. The capacity increases most efficiently by increasing parameters outside the logarithmic function. Therefore, the number of WDM channels is very important, meaning that a good comb source should have a broad bandwidth with many separate lines. The number of SDM channels and the modulation rate scales the same way, but where the modulation rate has an upper limit given by state-of-the-art electronics (today allowing for roughly $30 \mathrm{GHz}$ efficient modulation with high bit resolution), there is no obvious upper limit for SDM. We investigate the total capacity based on the SNR straight out of the comb as an instructive indication of the data carrying capacity of a comb source.
The analysis of the data carrying capacity of a single source is performed using a setup similar to that in Figure $2 \mathrm{~A}$, but where the comb is split in $m_{\mathrm{SDM}}$ branches just after the pre-amplifier, $G_{\text {pre. }}$. To compensate for the loss of the power splitting by the SDM-splitter, each SDM branch gets an additional amplifier, $G_{\mathrm{SDM}}$, which in part amplifies the signal to the desired level, but also introduces more ASE noise, and amplification of the noise prior to this step. Each branch then splits the wavelength channels into $n_{\text {WDM }}$ channels, with no loss, and each line is data modulated, with a polariser at the input to the modulator (effectively removing half the ASE noise). The modulator is assumed to be a dual-polarisation IQ-modulator using the signal power as efficiently as possible. All WDM lines are then recombined in a lossless WDM-multiplexer, and the loss of the whole modulator section is compensated for by an EDFA, $G_{\text {mod }}=15 \mathrm{~dB}$. After this step, all $m_{\text {SDM }}$ branches are recombined in a lossless power combiner and launched into the transmission link with $P_{\text {launch }}$ set to $0 \mathrm{dBm}$. So essentially the model is like Figure 2A with SDM multiplexing stages in between $G_{\text {pre }}$ and the link.

For $R_{s}=32 \mathrm{GBaud}, \mathrm{FSR}=35 \mathrm{GHz}$ and $300 \mathrm{WDM}$ channels (corresponding to $C$ and $L$-bands, the Shannon capacities for $\mathrm{m}$ cores shown in Figure 3A is obtained for five different line powers for a single link of $80 \mathrm{~km}$ length followed by an amplifier in the receiver. As seen, the total capacity scales with the number of SDM channels, and even though each comb line splitting to a new SDM channel decreases the available OSNR for that channel, the scaling continues favourably to 1000s of SDM channels. For instance, at just above 1000 SDM channels, a capacity of $100 \mathrm{Pbit} / \mathrm{s}$ is reached. And this is for realistic per comb lines powers of about -20 to $-10 \mathrm{dBm}$. So the data carrying capacity is really tremendous.

Figure 3B shows the Shannon capacity for a different case, where a mode-locked laser comb is used to seed a nonlinear chip to create a wider spectrum by self-phase modulation. In the study by $\mathrm{Hu}$ et al. [2], using an AlGaAs nano-waveguide, about 400 lines were created at $10 \mathrm{GHz}$ spacing with about $-15 \mathrm{dBm}$ power in each line [2]. If these 400 lines could all be modulated with $8 \mathrm{GBd}$, leaving some guardband, the total capacity for 30 spatial channels would be $1.18 \mathrm{Pbit} / \mathrm{s}$ after transmission through a $10 \mathrm{~km}$ piece of 30-core fibre with roughly $0.3 \mathrm{~dB} / \mathrm{km}$ loss. In the study by $\mathrm{Hu}$ et al. [2], however, as it was not possible to carve optical spectra with sharp enough filter shapes to separate $10 \mathrm{GHz}$ channels, instead four lines were used to make up one channel, and the $10 \mathrm{G}$ symbols were time domain multiplexed to match the $4 \times 10 \mathrm{GHz}$ lines with a $40 \mathrm{GBd}$ symbol rate. Every fifth line was suppressed as a 
A

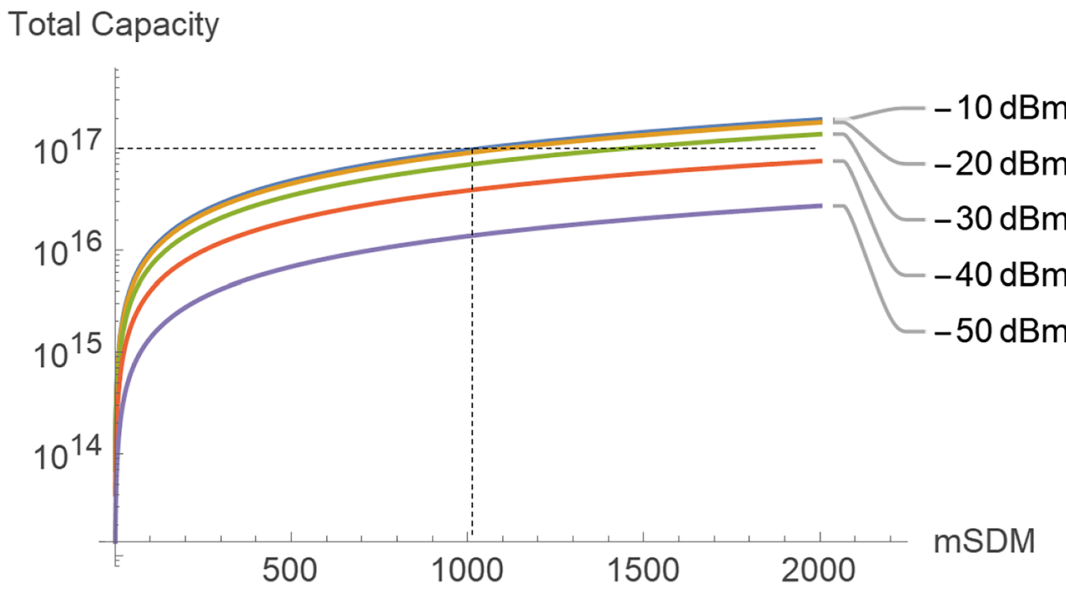

B

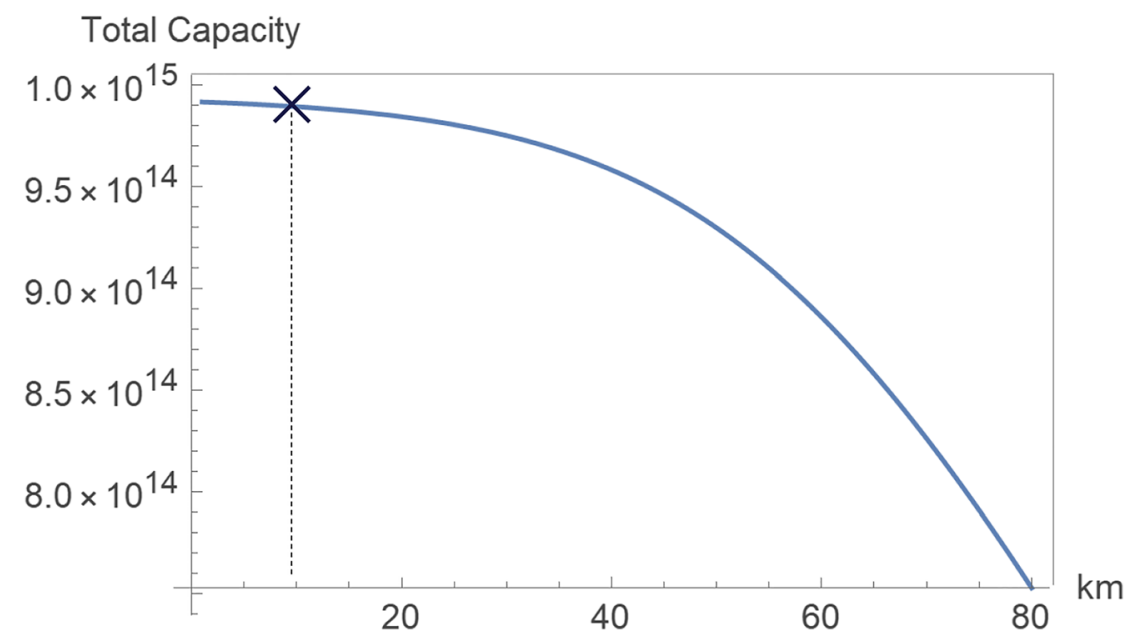

Figure 3: (A) Capacity for SDM multiplexing. The capacity quickly scales to high numbers, $\mathrm{Pbit} / \mathrm{s}$ to $10 \mathrm{~s}$ of $\mathrm{Pbit} / \mathrm{s}$, and then starts to slowdown in growth as a result of diminishing OSNR from splitting the initial OSNR per comb line into many cores. However, a steady growth in capacity continues, and extreme rates can be obtained. For the parameter values used here, it is found that $100 \mathrm{Pbit} / \mathrm{s}$ may be reached with just over 1000 SDM channels and -20 to $-10 \mathrm{dBm}$ initial power per comb line, and $1 \mathrm{Pbit} / \mathrm{s}$ is reached for only 10-15 SDM channels for the same comb line powers. (B) The Shannon capacity calculated for a recent 30-core fibre transmission experiment using a comb source as in the study by Hu et al. [2]. A mode-locked laser with an initial comb of about 40 lines at $10 \mathrm{GHz} F S R$, power per comb line of $-6 \mathrm{dBm}$, and optical carrier to noise ratio of $50 \mathrm{~dB}$ is used as the seed for a self-phase modulation unit that broadens the comb to 400 lines. As described in the study by $\mathrm{Hu}$ et al. [2], these lines are grouped in four lines per channel, and using optical timedivision multiplexing to reach $40 \mathrm{Gbaud}$ $50 \mathrm{GHz}$ spaced WDM channels. With 80 such 40-Gbaud WDM channels in 30 cores, it was experimentally found that $661 \mathrm{Tbit} / \mathrm{s}$ could be successfully transmitted through a $10 \mathrm{~km}$ long fibre. guardband, leaving 80 WDM channels. The power per WDM channel is then $4 \times$ the power per $10-\mathrm{GHz}$ line, i.e. $-15 \mathrm{dBm}+6 \mathrm{~dB}=-9 \mathrm{dBm}$. For $30 \mathrm{SDM}$ channels the total capacity after a 30 -core fibre $10 \mathrm{~km}$ transmission becomes 989 Tbit/s. $661 \mathrm{Tbit} / \mathrm{s}$ were achieved in the experiment in the study by $\mathrm{Hu}$ et al. [2], due to available modulation capacity at the time, but nonetheless, this experiment and capacity calculation clearly demonstrates the high data carrying capacity of frequency combs.

\subsection{Energy efficiency}

It has been speculated that a comb source could save a lot of energy in transmitters relying on individual lasers for individual WDM channels. As we have seen in Figure 2 above, a comb source certainly does not produce the same amount of line power as an individual laser does, impairing the achievable OSNR. For long reach links, though, we saw that even for comb powers of less than $-20 \mathrm{dBm}$ the OSNR penalty was less than $1 \mathrm{~dB}$ for more than $1000 \mathrm{~km}$ transmission. For short reach, the combs start needing more power to compare to individual lasers. However, even for unrepeatered metro-range transmission, very decent OSNRs may still be obtained for combs, and $661 \mathrm{Tbit} / \mathrm{s}$ was experimentally demonstrated feasible over $10 \mathrm{~km}$ 30-core fibre. So combs can offer performance at a reasonable level. Especially, when multiplexing scenarios are considered as a large gain in capacity is obtained for terms outside the $\log _{2}$ in the capacity formula. But can combs be more energy-efficient than single lasers. It is difficult to compare mature commercial solutions to lab-setups. However, there certainly is a potential, as all the comb lines are equidistant and stable, so no additional wavelength locking is required 
on a per channel basis, and a fewer current sources and laser temperature stabilizers are needed. As mentioned above, this also gives rise to certain digital signal processing benefits, such as joint DSP and nonlinear impairment mitigation. On the other hand, a comb needs a strong pump signal, and amplification of the comb lines, which costs energy. But assuming e.g. replacing $100 \mathrm{~s}$ of lasers with a single comb source, as potentially done for the hero experiment reaching $10 \mathrm{Pbit} / \mathrm{s}$ transmission [27] using a single source, with all their power sources etc., and knowing that the comb can perform well in some ranges, we expect there would be a considerable savings potential. One essential parameter in this consideration is the pump to line conversion, as this directly impacts the achievable OSNR. This depends on the nonlinear material used, the scheme of comb-generation, and losses in the device.

\section{Integrated mode-locked laser combs}

Mode-locked lasers generate short pulses with fixed pulse repetition rate and an optical frequency comb spectrum with a frequency spacing equal to the repetition rate. Semiconductor-based integrated mode-locked lasers (MLL) are attractive with respect to compactness, robustness, performance stability, low power consumption and low cost [28]. Optical frequency combs generated by MLL are electrically pumped, and hence do not need an additional pump laser, as some other comb sources, making them energy-efficient and easier to fully integrate with a small form factor. Different with fibre-based MLL, integrated MLL can work at fundamental mode and avoid supermode noise, however, optical linewidth of integrated MLL is typically too high to be used for coherent communications. In order to reduce the optical linewidth, laser cavity needs to be extended.

Mode-locking can be categorized into passive, active and hybrid mode-locking [28-34]. Passive mode-locked lasers have the advantage of simple operation, merely being driven by a DC current. A typical passive modelocked laser consists of a saturable absorber and a gain medium in a laser cavity. The saturable absorber (SA) is an optical intensity-dependent component, which has a decreasing loss with increasing light intensity. When the electrical current injected into the gain medium is above a certain threshold, the laser modes start to oscillate in noisy bursts with random phases. If one of these bursts has enough energy to make the SA start to saturate, the peak of the burst will have less loss, whereas the low-intensity wings of the burst will have more loss. When the most energetic spike travels back and forth in the laser cavity, the SA will selectively let the high-intensity spike pass and attenuate the low-intensity light. After many round trips until a steady state is reached, the laser starts to emit a train of phase-coherent mode-locked pulses. The passive modelocked laser can also work on hybrid mode locking by injecting an external RF electrical signal to the saturable absorber with the frequency of the injected RF signal being close to the repetition rate of the free-running passive mode-locked laser. When the laser works in hybrid modelocking, the phase noise of the laser can be stabilized and the frequency spacing of the mode-locked laser comb can be fine-tuned within a certain range.

The dynamics of absorption and gain of a mode-locked laser play a crucial role. At an initial state, the unsaturated loss is higher than the gain. When a burst occurs and the leading edge of the spike reaches the $\mathrm{SA}$, the loss saturates and becomes lower than the gain, which leads to a net gain window. Then, the SA recovers from the saturation state to the initial state of higher loss than the gain, thus attenuating the trailing edge of the spike. The recovery time of the SA and the saturation threshold are relevant for the generation of the mode-locked pulses. The SA can be monolithically integrated into a semiconductor laser, as shown in Figure 4A. By increasing the reverse bias on the SA section, the generated carriers can be more efficiently swept out of the absorber, resulting in a faster recovery time. From a materials point of view, quantum dots and dashes (QD) can have faster recovery times and lower saturation thresholds than quantum wells, leading to shorter pulse generation and reduced intrinsic noise [28]. Usually, a quantum well-based mode-locked laser frequency comb has a limited bandwidth of a few nanometres [33]. QD materials have homogeneously broadened gain spectrum, which can be used to generate a broader modelocked laser frequency comb with a bandwidth of $10 \mathrm{~nm}$ and above [28], and with per comb line powers in the useful range around $-15 \mathrm{dBm}$, as shown in Figure 4B.

\subsection{Quantum dot and quantum dash mode- locked lasers on InP substrates}

Typically, quantum dots and quantum dashes are grown by chemical beam epitaxy (CBE) or molecular beam epitaxy (MBE) on InP substrates [30, 35]. Liu et al. [30] demonstrated a quantum dash passively mode-locked laser (MLL) frequency comb with a frequency spacing of $\sim 34 \mathrm{GHz}$, driven by an electrical direct current (DC). Figure $5 \mathrm{~A}$ shows the cross-sectional scanning electron microscopy (SEM) 
A

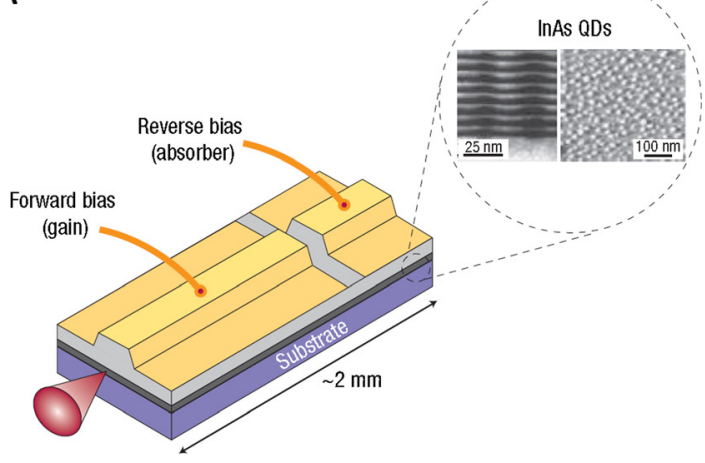

B

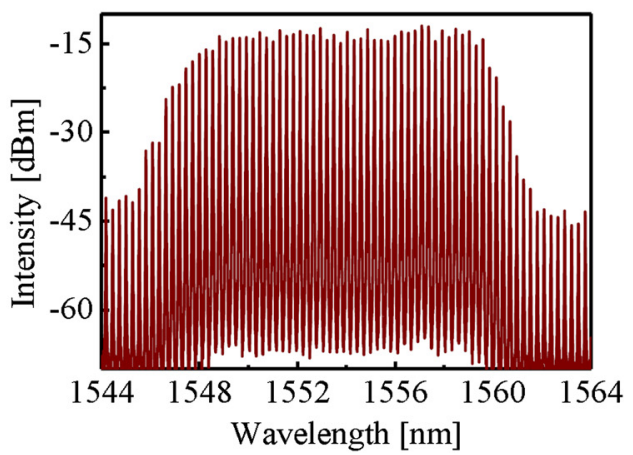

Figure 4: An integrated device and a MLL-based frequency comb.

(A) Schematic of chip-based passive mode-locked laser with integrated gain and absorber, (inset) TEM photographs of quantum dots layers [28]. (B) Optical spectrum of a QD-MLL-based frequency comb [30]. Reprinted with the permissions from Ref. [28] Copyright 2007, Springer Nature and Ref. [30], Copyright 2020, Optical Society of America.

image, showing the facet of a fabricated Fabry-Perot (FP) InAs/InP quantum dash laser. The schematic crosssectional view of a shallow-etched ridge-waveguide InAs/ InP QD laser is shown in Figure 5B. The InGaAsP waveguide contains five stacked layers of InAs QDs as the gain medium, surrounded by $n$ - and $p$ - type InP cladding layers. The MLL frequency comb has a 6-dB bandwidth of $\sim 13 \mathrm{~nm}$ and 48 comb lines with an optical carrier to noise ratio (OCNR) of more than $40 \mathrm{~dB}$, as shown in Figure 4B. The average optical linewidth of each comb line is around $1.55 \mathrm{MHz}$, as shown in Figure 5B. The MLL frequency comb was used for a PAM-4 signal transmission over a $25-\mathrm{km}$ standard single mode fibre (SSMF), with an aggregated capacity of $5.376 \mathrm{Tbit} / \mathrm{s}$ [30]. By modulating with a 16-QAM signal, the MLL frequency comb was also used for a 10.8 Tbit/s transmission over a 100-km SSMF [35]. In addition, a MLL frequency comb can be used not only as a laser source in a transmitter, but also as a local oscillator in a coherent receiver [36], though the linewidth would need to be sub-100 kHz.

The current limitation of using integrated MLL-based frequency combs for large capacity optical communication systems is still the relatively broad optical linewidth, which is on the order of $1-20 \mathrm{MHz}$ and at least an order of magnitude broader than most external cavity lasers (ECL), widely used in coherent transmission systems today. The optical linewidth of an MLL frequency comb limits the modulation format useable in transmission experiments to mainly intensity modulation, such as pulse amplitude modulation (PAM) signals, or simple phase modulation formats, such as QPSK or 16-QAM with degraded performance [30, 35, 37]. Nevertheless, several schemes have been
A

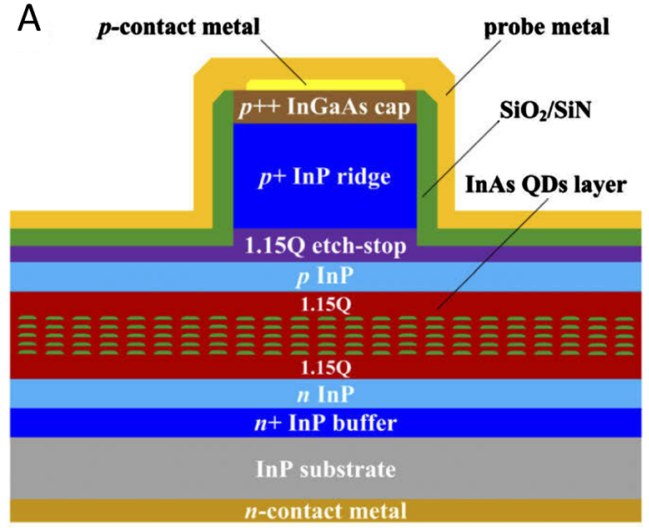

B

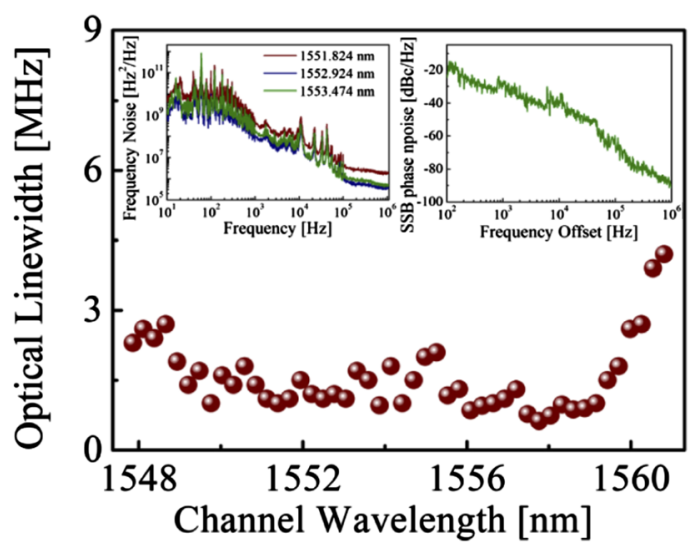

Figure 5: A passively mode-locked InAs/InP quantum dash laser.

(A) Schematic of the cross section. (B) Optical linewidth of filtered individual comb lines. (Left inset) frequency noise spectra from three filtered comb lines at the wavelengths of $1551.824,1552.924$ and $1553.474 \mathrm{~nm}$ (Right inset) Single sideband (SSB) phase noise of the RF beating. Reprinted with the permission from Ref. [30], Copyright 2020, Optical Society of America. 
proposed to solve this problem, such as introducing an external cavity to reduce the optical linewidth [38] or using a digital phase tracking algorithm to improve the transmission performance [39]. Kemal et al. [38] built a free-space external cavity for a QD-MLL reducing the optical linewidth by almost two orders of magnitude to $\sim 40 \mathrm{kHz}$, which was then used for 11.2 Tbit/s 32-QAM signal transmission over a $75 \mathrm{~km}$ SSMF. As a fully integrated alternative to free-space optical external cavity lasers, low-loss silicon waveguides have been hybridly integrated with a laser cavity to enable a III-V-on-Si MLL frequency comb with an optical linewidth of $<400 \mathrm{kHz}$ [40], which looks very promising.

\subsection{Quantum dot mode-locked lasers directly grown on $\mathbf{S i}$}

Recent breakthroughs on direct growth of quantum dot materials on CMOS-compatible silicon substrates provide an appealing direction from both cost and performance perspective, as it combines the advantages of active quantum dot materials with the mass-production ability of silicon [41]. Liu et al. [42] demonstrated a 20-GHz passively mode-locked quantum dot laser which is directly grown on an on-axis (001) CMOS-compatible silicon substrate, with the schematic shown in Figure 6A. The laser operates in the $\mathrm{O}$ band with a 3-dB bandwidth of $6.1 \mathrm{~nm}$ and 80 comb lines within the 10-dB bandwidth. The average optical linewidth of each comb line is around $10.6 \mathrm{MHz}$, as shown in Figure 6B. The QD-MLL-on-Si frequency comb was used as the WDM light source for a terabit PAM-4 signal transmission over a $5-\mathrm{km}$ SSMF.

\section{Spectral broadening of frequency combs}

MLL-based frequency combs typically have a comb bandwidth of $<20 \mathrm{~nm}$, so if e.g. a full C-band comb is desired, further broadening of the spectrum is required. This may be achieved by nonlinear spectral broadening, such as selfphase modulation (SPM)-based spectral broadening. Here, short pulses passing through a third-order $\left(\chi^{(3)}\right)$ nonlinear medium with slightly negative dispersion undergo a selfphase modulation process, resulting in a large chirping of the pulses and a spectral broadening, as illustrated in Figure 7A. With a sufficiently strong interaction and suitable dispersion in the waveguide, the bandwidth of the broadened frequency comb can exceed an octave, whilst maintaining the FSR of the individual comb lines, and yielding very high per comb line power. Highly nonlinear fibre (HNLF) is one attractive solution for the nonlinear material to create wideband high-power frequency combs spectral broadening, and has been used for optical communications in several high-capacity experiments $[27,43$, 44]. Typically tens to hundreds of metres of HNLF is required, making it incompatible with full on-chip integration, which may be useful in some scenarios. Integrated waveguides of various material platforms with high nonlinearity have been demonstrated for supercontinuum generation (SCG), and octave-spanning chip-based SCG has been reported [45-51]. In a silicon waveguide, octavespanning (1.5-3.3 $\mu \mathrm{m}) \mathrm{SCG}$ was demonstrated using 70-fs pump pulses with a pulse energy of $16 \mathrm{pJ}$ [46]. The pump wavelength is in the mid-infrared region at $2.29 \mu \mathrm{m}$, which is high enough to avoid the strong two-photon absorption (TPA) in silicon. On a TPA-free material platform, pump pulses can be placed in the telecom $\mathrm{C}$ band for the SCG. Using pump pulses with a wavelength around $1.55 \mathrm{um}$, SCG over more than two octaves spanning from 0.56 to $3.6 \mu \mathrm{m}$ was demonstrated in a silicon nitride $\left(\mathrm{Si}_{3} \mathrm{~N}_{4}\right)$ waveguide with a pulse energy of $\sim 1 \mathrm{~nJ}$ [50], and SCG spanning from 0.4 to $2.4 \mu \mathrm{m}$ was demonstrated in a lithium-niobate waveguide with a pulse energy of $\sim 180 \mathrm{pJ}$ [47]. Very broad bandwidth SCG spectra are useful for many applications, such as spectroscopy and frequency metrology. However, for optical communications, covering the telecom $\mathrm{C}+\mathrm{L}$ bands $(\sim 80 \mathrm{~nm})$ is often sufficient, therefore, SPM-based
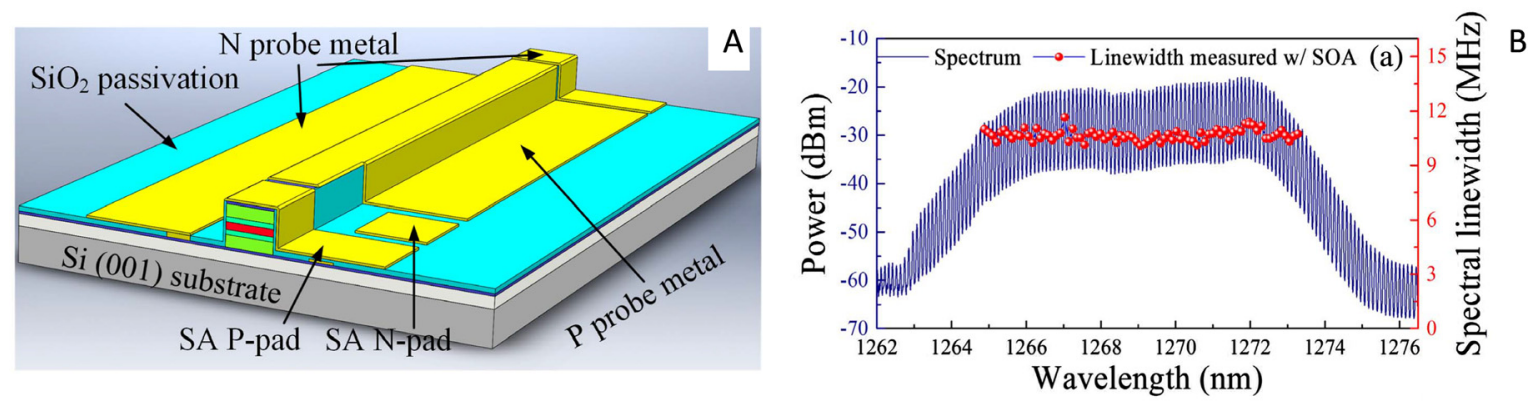

Figure 6: (A) Schematic of the $20 \mathrm{GHz}$ quantum dot passively mode-locked laser on silicon (not to scale). (B) Optical spectrum and optical linewidth of individual comb lines. Reprinted with the permission from Ref. [42], Copyright 2019, Optical Society of America. 

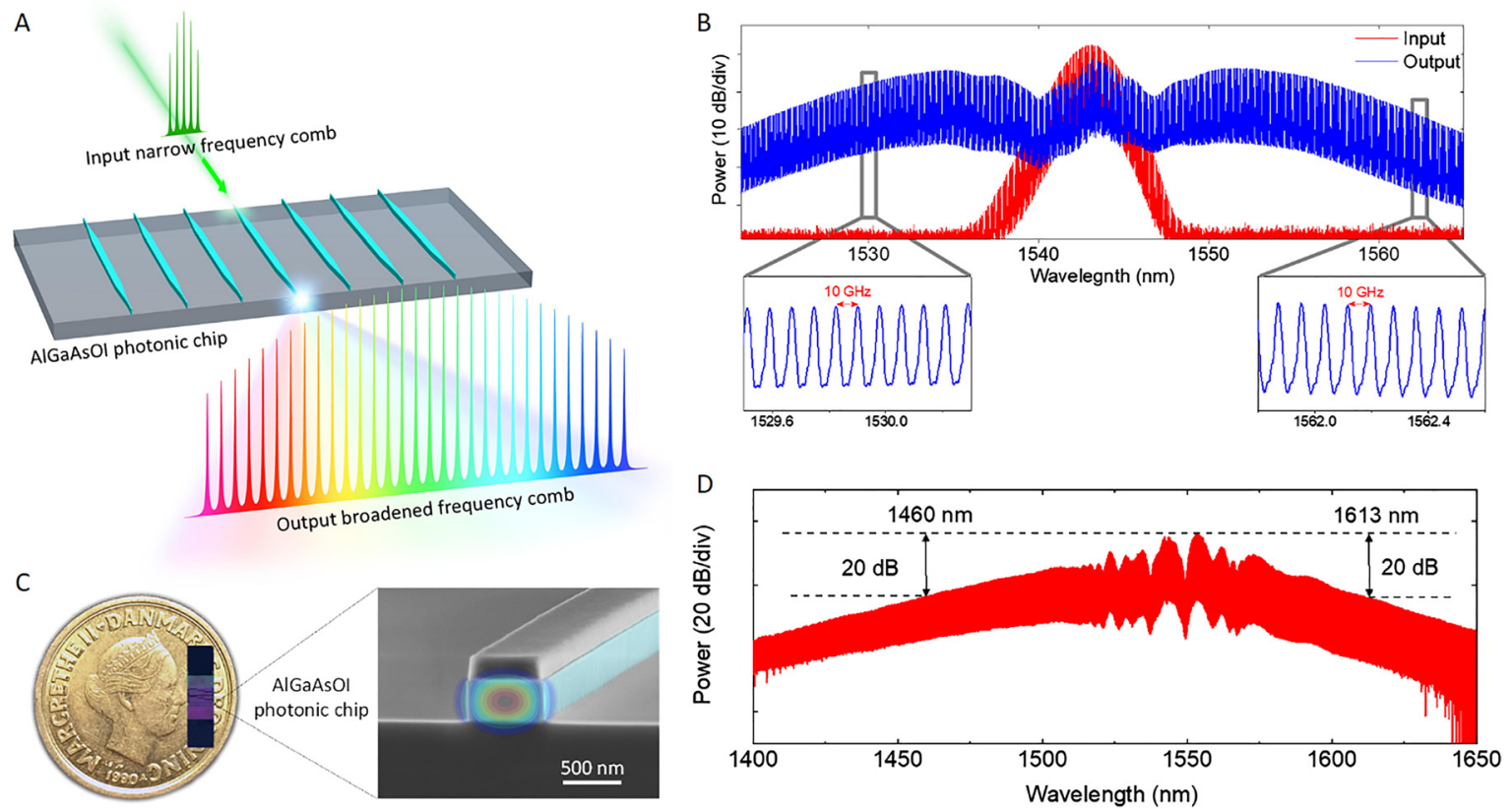

Figure 7: (A) Schematic of spectral broadening of frequency comb on an AlGaAsOI nano-waveguide. (B) Optical spectra of the 10-GHz 1.5-ps seed pulses and generated broadband frequency comb. The input frequency comb (20-dB bandwidth of $6.4 \mathrm{~nm}$ ) is spectrally broadened to a $20-\mathrm{dB}$ bandwidth of $\sim 44 \mathrm{~nm}$, covering more than the telecom $C$ band. The comb lines are equidistantly spaced by $10 \mathrm{GHz}$, same as the repetition rate of the seed pulses. (C) The AlGaAsOI photonic chip is smaller than a coin, but can accommodate hundreds of nano-waveguides. The SEM image shows the cross section of the nano-waveguide with cross-section dimensions of $280 \times 600 \mathrm{~nm}$, indicating strong light confinement (artificially added: the simulated field distribution for the fundamental TE mode). (D) Optical spectrum of the generated frequency comb using $10 \mathrm{GHz}$ fs pulses (FWHM of $230 \mathrm{fs}$ ) as the seed. (The figures are adapted from the study by Hu et al. [2]).

spectral broadening of frequency combs with low noise is desirable in this scenario.

The ultra-broad SCG demonstrations mentioned above are all achieved with low repetition rate $(<1 \mathrm{GHz})$ femtosecond pulse trains. For optical communications, repetition rates in the range $10-100 \mathrm{GHz}$ is often desired. The peak power of such pulses is much lower than that of femtosecond pulses at low repetition rates, so an ultra-efficient nonlinear platform is needed for spectral broadening of frequency combs. The low loss and high nonlinearity characteristics of the AlGaAs-on-insulator (AlGaAsOI) waveguide make it a desirable material platform. Spectral broadening of frequency combs with $10-\mathrm{GHz}$ frequency spacing is achieved in an AlGaAsOI nanowaveguide, using picosecond pump pulses in the telecom $C$-band with a peak power of only $\sim 5.6 \mathrm{~W}$ [2]. Note that the bandgap of AlGaAs can be engineered by changing the $\mathrm{Al}$ concentration to minimise nonlinear absorption at telecom wavelengths [52-55]. The bandwidth of the $10 \mathrm{GHz}$ broadened frequency comb in the study by Hu et al. [2] is $\sim 44 \mathrm{~nm}$, which can cover more than the telecom $\mathrm{C}$ band, as shown in Figure 7B. The broadened frequency comb in the AlGaAsOI photonic chip (Figure 7C) was data modulated with
16-QAM and used to demonstrate $661 \mathrm{Tbit} / \mathrm{s}$ net-rate data transmission over a single-mode 30-core fibre [2]. When the pump pulses were compressed to femtosecond pulses with a pulse width of $230 \mathrm{fs}$, the broadened frequency comb with a bandwidth of $153 \mathrm{~nm}$ (from 1460 to $1613 \mathrm{~nm}$ ) was achieved, which can cover $S, C$ and $L$ band, as shown in Figure 7D.

The broadened frequency comb in the non-resonant nano-waveguide is insensitive to temperature change, making it resilient to environmental changes and facilitates long-term stability. If the seed mode-locked laser is hybrid mode-locking, which can be synchronized to an external clock, the frequency spacing of the frequency spacing of the comb can be controlled with high accuracy [2]. For a coherent broadened frequency comb, the linewidth of the generated comb lines can be close to that of the seed laser for a broad range [56], save for possible added phase noise in the nonlinear process. The pump-to-comb conversion efficiency is defined as the ratio between the power of the pump to that of the generated comb lines. The spectral broadening of frequency comb has the advantage of high pump-to-comb conversion efficiency, which allows for sufficient per comb line power at the chip output and 
enables low energy consumption of the frequency comb. Sixty six percentage pump-to-comb conversion efficiency was demonstrated in the study by $\mathrm{Hu}$ et al. [2], where the comb was used to carry $661 \mathrm{Tbit} / \mathrm{s}$ through a 30-core fibre. The per comb line power was $-15 \mathrm{dBm}$, and as discussed in Section 2.3 and Figure 3 (B), this was enough to carry this large amount of data.

\section{Microresonator-based Kerr frequency combs}

Unlike spectral broadening of frequency combs seeded by short pump pulses, microresonator-based Kerr frequency combs (often called micro-combs) are typically pumped by a single-frequency continuous-wave (CW) pump beam. In the process of micro-comb generation, the $\mathrm{CW}$ pump can produce gain for microresonator cavity modes through cascaded four-wave mixing (FWM), and when optimal conditions are met (sufficiently strong nonlinearity and appropriate dispersion), a micro-comb is generated with a frequency spacing given by the free spectral range (FSR) of the microresonator. Micro-combs have been intensively investigated recently and exhibits rich and complex nonlinear behaviours [3,57-63], including chaos, single and multiple soliton formation, bright and dark soliton, soliton crystals and breather instabilities [64-67]. The dynamics of the micro-comb is well described by the Lugiato-Lefever equation [68-71]. The micro-comb generation has been observed in a number of chip-based platforms including silicon nitride [8, 72-78], Hydex glass [73, 79], silica [80, 81], silicon [82, 83], diamond [84], aluminium nitride [85, 86], aluminium gallium arsenide [52, 53], gallium phosphide [87] and lithium niobate $[88,89]$. In an early-stage study, a micro-comb operating in a chaotic state was used as the WDM source for optical communications with a data rate of $1.44 \mathrm{Tbit} / \mathrm{s}$, limited by incoherent phase and strong irregularities of the optical spectrum [90]. Using dissipative Kerr soliton (DKS) micro-combs give much better results for communications, yielding micro-comb-based systems with capacities exceeding $10 \mathrm{Tbit} / \mathrm{s}$ based on so called bright DKS, dark pulses and soliton crystals [91-93]. Note that, micro-combs can also be pumped by pulses [61, 94], which exhibit improved pump-to-comb conversion efficiency and tunability of pulse repetition rate.

\subsection{Bright dissipative Kerr soliton micro-combs}

A soliton is a waveform that maintains its shape while propagating, upheld by a balance of dispersion and nonlinearity in the medium $[95,96]$. The dissipative Kerr soliton (DKS) relies on a double balance of parametric gain and cavity loss, as well as dispersion and nonlinearity. Figure 8A shows the schematic setup of DKS micro-comb generation in a high- $Q$ silicon nitride $\left(\mathrm{Si}_{3} \mathrm{~N}_{4}\right)$ microresonator [91]. The integrated micro-ring resonator is pumped by a tunable CW laser which is amplified by an erbium-doped fibre amplifier (EDFA). At the output of the microresonator, a notch filter is used to suppress the remaining pump light. In order to generate the bright DKS micro-comb, the pump laser is swept through the resonance from a blue-detuned wavelength to a predefined reddetuned wavelength [97-99]. Figure 8B shows the single soliton and multiple solitons. When the pump laser is tuned across the cavity resonance, high-noise modulation instability (MI) combs are first observed. When the pump laser is tuned backwards, bistability of the cavity enables the formation of soliton states from a multiple-soliton state to a single-soliton state. The power spectrum of the DKS comb state is shown in Figure $8 \mathrm{C}$ and exhibits a 3-dB spectral bandwidth of $\sim 6 \mathrm{THz}$. The DKS comb states are stable for many hours in a laboratory environment, which is key for using the sources in optical transmission experiments. The pump-to-comb conversion efficiency of the DKS micro-comb is limited to $0.1 \%-0.6 \%$ owing to the fundamental principle that bright DKS generation occurs only with the pump laser being far-detuned from the resonance.

The DKS micro-combs are used in three optical transmission experiments [91]. In the first experiment, the DKS micro-comb with $\sim 100 \mathrm{GHz}$ spacing is modulated with 16-QAM signals and the data with an overall net rate of $28 \mathrm{Tbit} / \mathrm{s}$ is transmitted on 94 carriers spanning telecom $C$ and $L$ bands. In the second experiment, the number of carriers is doubled by interleaving two DKS micro-combs. The overall net rate of $50.2 \mathrm{Tbit} / \mathrm{s}$ is achieved with a total of 179 carriers. In the third experiment, the DKS micro-combs are used both as the source in the transmitter and as the multi-wavelength local oscillator in the coherent receiver. This is a benchmark result, clearly showing the potential of these combs. In these experiments, the pump power to the ring was $32 \mathrm{dBm}$, but this can be lowered with further optimization of the losses of the ring.

Furthermore, a pump source integrated with a microring has been demonstrated on an integrated hybrid III-V/ $\mathrm{Si}_{3} \mathrm{~N}_{4}$ platform, relying solely on supplying the pump source with a current from a standard AAA battery, thus ensuring low-power operation [100]. In this demonstration, a gain section based on a III-V reflective semiconductor optical amplifier (RSOA) is coupled to a $\mathrm{Si}_{3} \mathrm{~N}_{4}$ laser cavity, which consists of two Vernier microring filters 
A

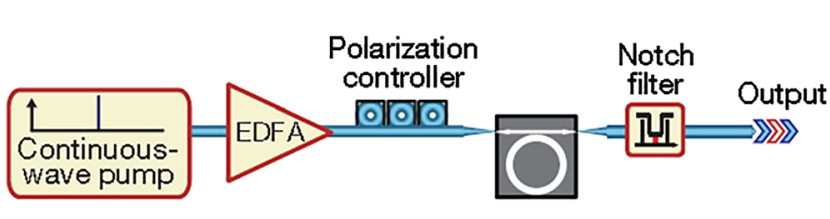

B

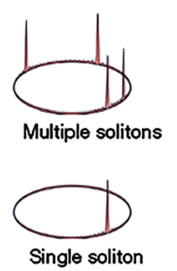

C

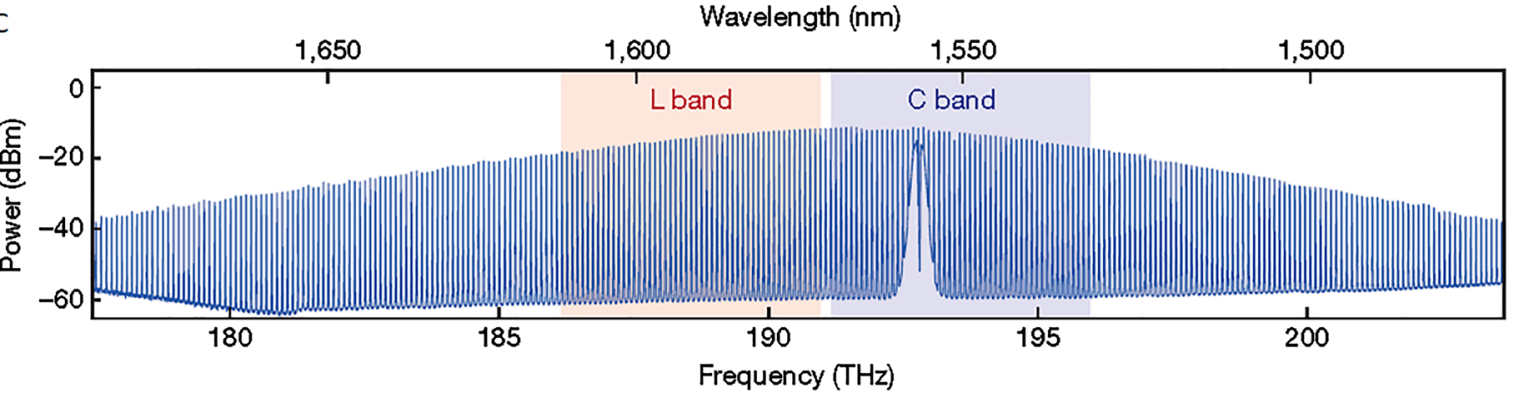

Figure 8: Bright DKS-based micro-comb generation in high- $Q$ silicon nitride microresonators.

(A) Schematic setup of the bright DKS micro-comb generation. (B) Illustration of single soliton and multiple solitons. (C) Optical spectrum of a single-soliton frequency comb after the suppression of residual pump light. Reprinted (adapted) with the permission from Ref. [91], Copyright 2017, Springer Nature.

for wavelength tunability and a high- $Q$ nonlinear microresonator. In order to achieve long-term stable operation, active feedback circuits are needed, which may be integrated together in future work.

Very recently, a turnkey operation regime for soliton micro-combs co-integrated with a pump laser has been demonstrated [102]. Solitons are immediately generated by turning the pump laser on when the device is working at an appropriate operating point, therefore, the photonic and electronic control circuitry is not needed. In the experiment, integrated soliton micro-combs with CMOS-compatible fabrication [101] and frequency spacing at electroniccircuit rates (40 GHz down to $15 \mathrm{GHz}$ ) are butt-coupled to a commercial distributed-feedback (DFB) laser via inverse tapers. Due to its compact footprint and the absence of control electronics, the pump laser and micro-comb chipset is mounted into a butterfly package (Figure 9A) to facilitate measurements and enable portability. The demonstrated turnkey operation is automatic, and the entire soliton initiation and stabilization is realized by the physical dynamics of laser self-injection locking in combination with the nonlinear resonator response. Therefore, the turnkey DKS micro-comb is highly robust with respect to temperature and environmental disturbances. Figure 9B shows the optical spectrum of a single-soliton state with a repetition rate of $40 \mathrm{GHz}$, and multi-soliton states with repetition rates of 20 and $15 \mathrm{GHz}$ are also demonstrated.

As mentioned in the previous section, the pump-tocomb conversion efficiency is an important metric. The pump-to-comb conversion efficiency of bright DKS microcombs has a theoretical limit of $5 \%$, as bright-soliton generation only occurs when the pump laser is detuned from the optical resonance $[61,99,103]$. Nevertheless, the overall pump consumption of the bright DKS micro-comb can already compete with massively parallel arrays of commercially available integrated tunable lasers [91], and
A

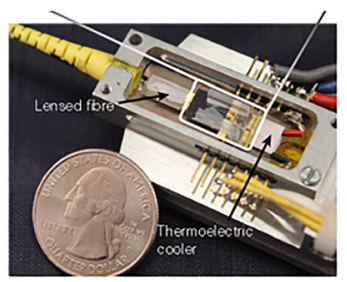

B

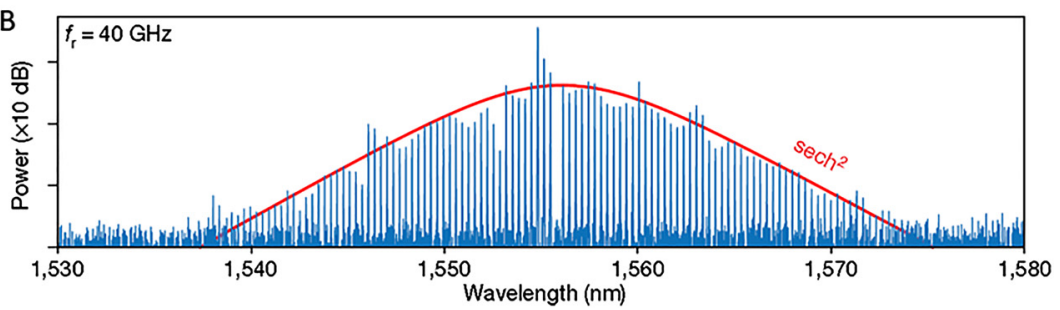

Figure 9: Integrated turnkey soliton micro-comb with the repetition frequency at electronic-circuit rates.

(A) Image of the DFB pump laser and micro-comb chipset mounted in a compact butterfly package. (B) Optical spectrum of a single soliton state with the repetition frequency of $40 \mathrm{GHz}$. Reprinted (adapted) with the permission from Ref. [102], Copyright 2020, Springer Nature. 
as mentioned in Section 2.3, per comb line powers on the order of $-20 \mathrm{dBm}$ suffice for many cases.

\subsection{Dark pulse micro-combs}

In contrast to bright soliton micro-combs, which are generated in anomalous dispersion microresonators, darkpulse Kerr combs (sometimes referred to as dark solitons) are generated in normal dispersion microresonators. Since the initial stage of micro-comb generation is triggered by modulation instability, normal dispersion has often been thought as unsuitable for micro-comb generation due to the lack of modulation instability. Nevertheless, modulation instability can occur in normal dispersion microresonators with the assistance of mode coupling [103-105], and mode-locked dark pulses can be generated. The darkpulse micro-comb is e.g. initialized by tuning the CW pump wavelength into the resonance from the blue side and a feedback loop ensures long-term stable operation. In the feedback loop, a part of newly generated comb line is filtered and detected by a photodiode and the output of the photodiode is used as feedback to the laser wavelength setting, therefore, the pump laser will stop sweeping when the frequency comb is generated. A spectrum of a darkpulses micro-comb is shown in Figure 10A, which operates in a low-noise state with a measured frequency spacing of 229 GHz. The dark pulses can be understood as time domain low-intensity dips in a high-intensity homogeneous background (Figure 10B), which exhibits unique advantages such as higher pump-to-comb conversion efficiency and flatter comb spectrum.

In a recent experimental investigation [25], a dark-pulse micro-comb reached $\sim 20 \%$ pump-to-comb conversion efficiency with weakest comb line power of $-10 \mathrm{dBm}$, and the comb lines were subsequently modulated with 64-QAM data and used in an optical transmission experiment with an aggregated data rate of $4.4 \mathrm{Tbit} / \mathrm{s}$. In a very recent experiment, a dark-pulses micro-comb with $105.2 \mathrm{GHz}$ spacing and covering telecom $C+L$ bands was modulated by probabilistically shaped 64 or 256 QAM and transmitted over a 37-core fibre, with an aggregated net rate of $1.84 \mathrm{Pbit} / \mathrm{s}$ [93].

\subsection{Soliton crystal micro-combs}

Soliton crystal micro-combs introduce a new regime of soliton physics into the field of micro-combs $[64,106]$, which are defined as spontaneously and collectively ordered ensembles of co-propagating solitons that are regularly distributed in a resonator. The co-propagating solitons interact with each other and "crystallize" by arranging themselves into a self-organized sequence. Soliton crystal micro-combs have a distinctive 'fingerprint' optical spectrum arising from spectral interference between interacting solitons. Since the solitons are tightly packed within the resonator, the intra-cavity optical power of the soliton crystals is very close to that of spatiotemporal chaotic states. There is very little change in intra-cavity power during the transition from chaotic states to soliton crystal states, therefore, soliton crystals can be stably formed using slow tuning techniques without the need for complex dynamic pumping and stabilization schemes. Soliton crystal microcombs have the unique advantages of intrinsic stability and high pump-to-comb conversion efficiency (due to large overlap between the CW pump and the tightly packed multiple solitons), although the overall spectrum is unevenly shaped with line-by-line intensity variations.

Figure 11A illustrates the single temporal defect crystal state used in a recent experiment [92]. To generate the soliton crystal micro-comb, the pump laser is automatically tuned to a pre-set wavelength. The primary comb (Turing pattern) is initially generated as the pump laser is tuned into resonance, and then the soliton crystals are formed in the micro-cavity with appropriate mode crossing, and a characteristic spectrum, as shown in Figure 11B. Figure 11C demonstrates the stability and repeatability of the soliton crystal micro-comb generation by showing a variation in

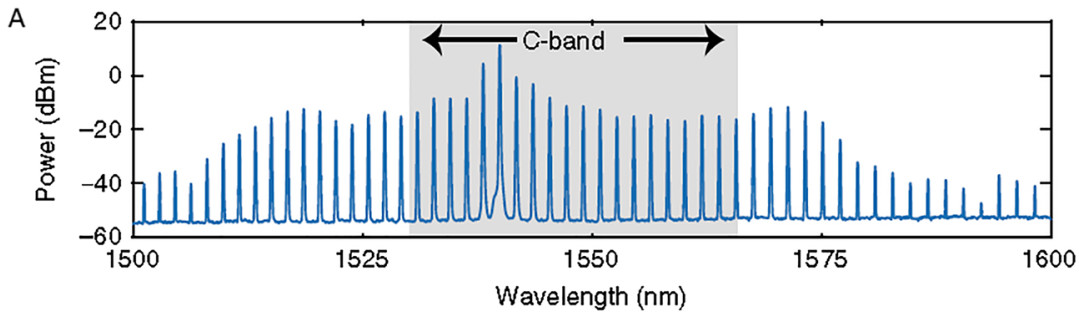

B

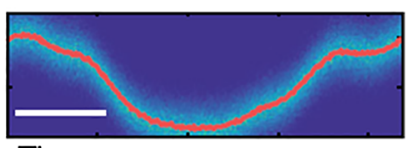

Time

Figure 10: Dark pulse micro-comb.

(A) Optical spectrum of the dark pulse micro-comb. (B) Directly measured intra-cavity time-domain waveforms. Reprinted (adapted) with the permission from Ref. [25], Copyright 2018, Springer Nature. 
individual tone powers of $< \pm 0.9 \mathrm{~dB}$ over 10 different generation instances through wavelength sweeping. The generated soliton crystal micro-comb in the study by $\mathrm{He}$ et al. [88] has a frequency spacing of $48.9 \mathrm{GHz}$ and a bandwidth $>80 \mathrm{~nm}$, with a high pump-to-comb conversion efficiency of $42 \%$. Eighty comb lines were selected over the telecom $C$ band and used as the WDM source in an optical data transmission experiment. A line rate of $44.2 \mathrm{Tbit} / \mathrm{s}$ and a high spectral-efficiency was achieved using the modulation format 64-QAM [92].

\section{Electro-optic frequency combs}

The above-mentioned chip-based frequency combs rely on either mode-locked lasers or third-order $\left(\chi^{(3)}\right)$ Kerr nonlinearity. An alternative method of frequency comb generation is using electro-optic (EO) effects in materials with second-order $\left(\chi^{(2)}\right)$ nonlinearity [107-111]. In a conventional EO frequency comb generator, a CW light passes through cascaded phase and amplitude modulators [13], which often results in high comb power, flat spectral shape and flexible frequency spacing. However, the EO frequency combs usually consist of bulky components and have a limited frequency span, typically tens of comb lines over a few nanometres spanning.

Broader EO frequency combs can be generated using a recirculating loop or an optical resonator to increase the nonlinear EO interaction strength $[112,113]$. The comb lines are generated through electro-optic phase modulation by the $\chi^{(2)}$ effect, and the modulated light is looped in a resonator, so when the modulation frequency matches a harmonic of the resonator free spectral range (FSR), the generated optical sidebands by the phase modulation are circulated back through the modulator so more sidebands will be generated. In a low-loss resonator, the light passes through the phase modulator multiple times before being dissipated or coupled out, efficiently generating many comb lines with the spacing equal to the modulation frequency. The modulation index determines the strength of coupling between neighbouring frequency components after passing through the phase modulator. The overall flatness of the EO frequency comb strongly depends on the round-trip modulation strength and the optical resonator loss. Therefore, strong phase modulation and a high- $Q$ optical resonator are crucial for generating flat and broad EO frequency combs. Dispersion in the cavity is also relevant for the total comb bandwidth, which can introduce frequency-dependent phase shifts. Previous free-space or fibre-based EO frequency combs have limited bandwidth due to weak phase modulation and limited dispersion engineering [114].

Figure $12 \mathrm{~A}$ shows a micrograph of an integrated microring EO comb generator, which integrates an EO phase modulator in a microring resonator with an FSR equal to the modulation frequency, as presented in the study by Zhang et al. [112] using a thin-film lithium niobate photonic platform. This modulator-resonator features a large $\chi^{(2)}$ nonlinearity, ultralow loss optical waveguides, strong microwave and optical field overlap and ability of dispersion engineering. The generated EO frequency comb has over 900 comb lines spaced by $\sim 10 \mathrm{GHz}$, spanning $80 \mathrm{~nm}$, as shown in Figure 12B. The EO comb has a slop of $1 \mathrm{~dB} / \mathrm{nm}$, i.e. comb line power drops $20 \mathrm{~dB}$ when the newly generated comb line is $20 \mathrm{~nm}$ from the centre. For the microring EO comb generator, the power per comb line is dependent on highest tolerable input optical power, insertion loss and the comb bandwidth. The low-loss lithium niobate microring resonator has a loaded $Q$ of $\sim 1.5$ million, and is integrated with microwave electrode for efficient phase modulation. This EO comb generator has a high tolerance to modulation frequency detuning,
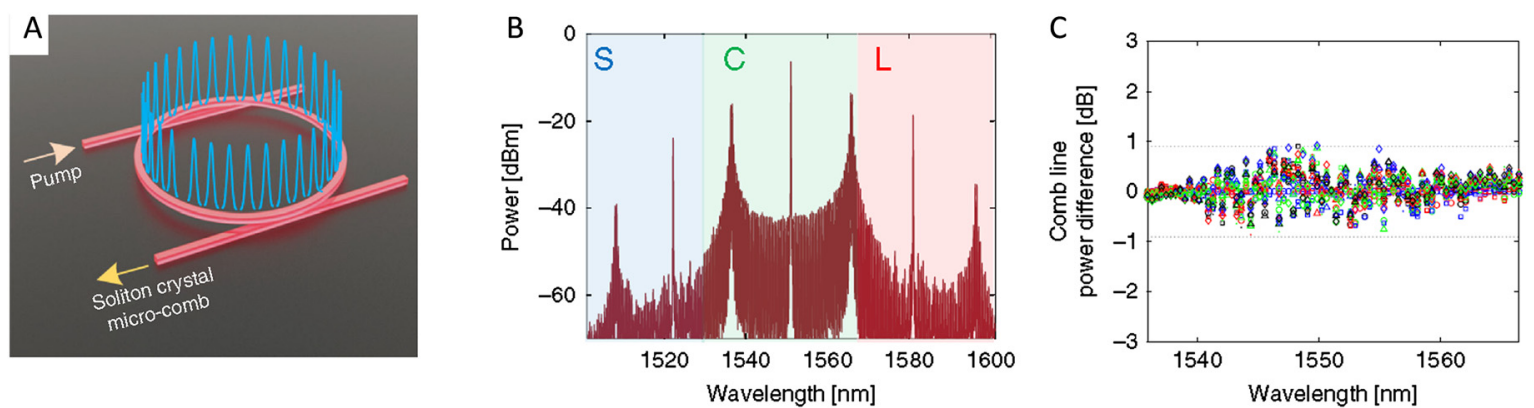

Figure 11: (A) Illustration of soliton crystal micro-comb state, which is a single temporal pulse defect across the ring. (B) Optical spectrum of the soliton crystal micro-comb. (C) The comb line power difference for 10 independent soliton crystal generation instances is within $\pm 0.9 \mathrm{~dB}$, indicating reliable generation of the soliton crystal micro-comb. Reprinted with the permission from Ref. [92], Copyright 2020, Springer Nature. 
A

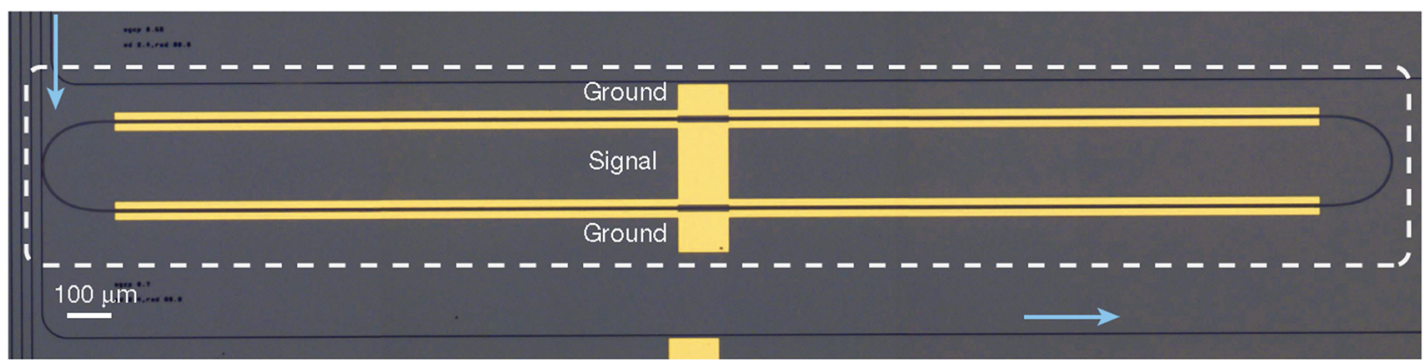

B

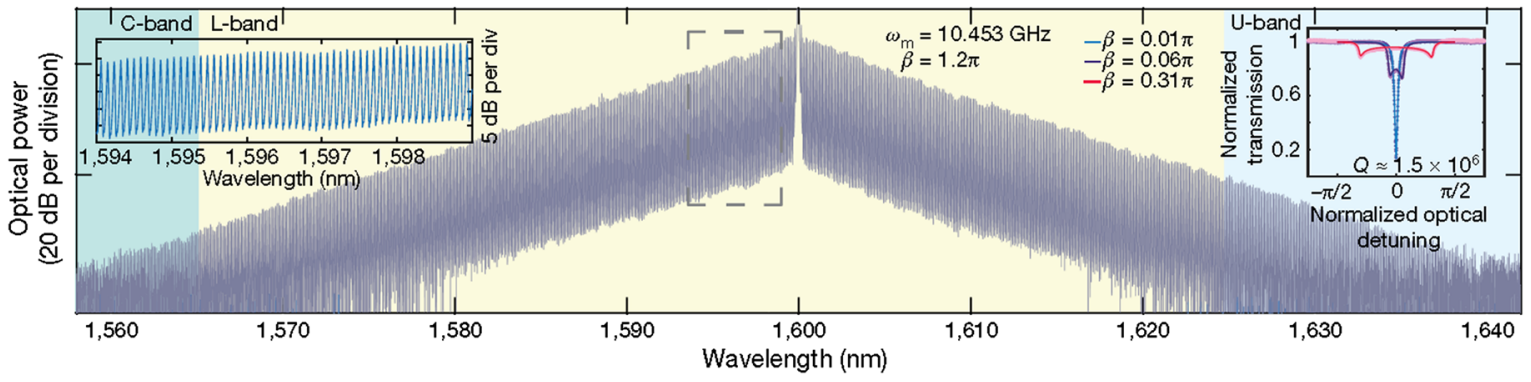

Figure 12: (A) Micrograph of an integrated lithium niobate microring resonator EO comb generator. (B) Measured optical spectrum of the EO frequency comb generated from the integrated lithium niobate microring resonator, with a bandwidth exceeding $80 \mathrm{~nm}$ and more than 900 comb lines with a slope of $1 \mathrm{~dB} \mathrm{~nm}^{-1}$. (Left inset) A magnified view of comb lines with a line-to-line power variation of $\sim 0.1 \mathrm{~dB}$ (Right inset) Measured transmission spectrum for different modulation indices. Reprinted with the permission from Ref. [112], Copyright 2019, Springer Nature.

allowing for a comb line frequency spacing finely tuned from $10 \mathrm{~Hz}$ to $100 \mathrm{MHz}$. In contrast, the frequency spacing of Kerr-based micro-combs is predetermined by the fabricated resonator dimensions [91]. The ability of tuning the frequency spacing of frequency combs is highly desirable for applications in optical communications, as it allows for alignment with the ITU (International Telecommunication Union) grid.

\section{Summary and discussion}

In this paper, we have reviewed recent achievements in chip-based frequency combs designed for use in optical communications, including mode-locked laser combs, spectral broadening of frequency combs, microresonatorbased combs and EO-modulated frequency combs, and their applications in high-speed optical communication systems. As opposed to applications such as spectroscopy and optical clocks, the optical communication applications do not require an octave bandwidth, and comb bandwidths of $10 \mathrm{~s}$ to $\sim 100 \mathrm{~nm}$ in the telecom $\mathrm{C}$ band or $\mathrm{C}+\mathrm{L}$ bands are usually enough. Characteristics of frequency combs for optical communications include absolute frequencies and frequency spacing aligned with the ITU-grid, low optical linewidth on the order of $10 \mathrm{kHz}$, adequate power per comb line to carry data on the order of -30 to $-10 \mathrm{dBm}$ or more, spectral flatness allowing for equalized signal power across the telecom band in question, and high pump-to-comb conversion efficiency. The two latter requirements indicate that the lowest comb line in a given bandwidth should not deviate too much from the average comb line power. One could otherwise achieve a high conversion efficiency, even though one would subsequently need to attenuate the high power comb lines to obtain and equalized spectrum of channels, i.e. throw away a lot of power, which would be undesirable. The first requirement suggests that tunability of centre frequency and frequency spacing is desirable. Mode-locked lasers can be thermally tuned to shift the centre frequency, and the frequency spacing can be tuned by an external RF clock, when it operates in hybrid mode locking. EO frequency combs may also be tuned by tuning the seed laser frequency and the resonance frequency together with the modulation frequency. Since spectral broadening of frequency combs are seeded by an optical pulse train, which can be derived from mode-locked lasers or EO combs, the centre frequency and frequency spacing are determined by the pulse train and can therefore also be tuned. The centre frequency of microrings is determined by the resonance frequency, which can be thermally tuned. However, the frequency spacing of the micro-combs mainly depends on the FSR of the fabricated microring resonator, which has limited tunability. 
Concerning optical linewidth, micro-combs and EO-based combs have linewidths determined by the CW pump laser, which can be a very narrow-linewidth laser. However, the optical linewidth of an integrated semiconductor mode-locked laser is typically on the order of several $\mathrm{MHz}$ to $10 \mathrm{MHz}$, making it unsuitable for a coherent detection optical communication systems. Nevertheless, direct detection optical communication systems usually do not require narrow optical linewidth sources, and integrated mode-locked lasers may therefore find applications in such systems. The optical linewidth of a MLL can be significantly reduced by hybrid integration with a low-loss external cavity [40], which could qualify such laser systems as useful for coherent communications. Spectral broadening of frequency combs may obtain narrow linewidths, if the seed pulse train has narrow linewidth. In term of energy efficiency, the spectral broadening of frequency combs show a high pump-tocomb conversion efficiency of $66 \%$, and the dark pulse micro-combs and soliton crystal micro-combs also show relatively high pump-to-comb conversion efficiency of $\sim 20$ and $42 \%$, respectively.

The power per comb line and spectral smoothness of the frequency comb can determine the OSNR of a frequency comb after the generated frequency comb is amplified by an optical amplifier. Higher power per comb line will result in higher OSNR of the frequency comb after the amplification. A high OSNR is usually required for the transmission of an advanced modulation format, such as high-order QAM. The power per comb line relies on the pump-to-comb conversion efficiency and the maximum launched pump power. On the other hand, high pump power requires the use of a high-power optical amplifier, which cannot be integrated on a chip. With the limited on-chip pump power, the pump-to-comb conversion efficiency will be the key to achieve a high power per comb line. In addition, the pump-to-comb conversion efficiency also indicates the overall power efficiency of the frequency comb. It is notable that several of the comb sources described here, have high enough comb line power to satisfy OSNR requirements in realistic systems, and hence be good value propositions for sources in transmitters for various range transmission systems with high capacities.

Acknowledgment: The authors would like to acknowledge funding support from the SPOC research center of excellence (DNRF123).

Author contributions: All the authors have accepted responsibility for the entire content of this submitted manuscript and approved submission.
Research funding: This research was funded by the SPOC research center of excellence (DNRF123).

Conflict of interest statement: The authors declare no conflicts of interest regarding this article.

\section{References}

[1] S. T. Cundiff and J. Ye, "Colloquium: femtosecond optical frequency combs,” Rev. Mod. Phys., vol. 75, no. 1, pp. 325-342, 2003.

[2] H. Hu, F. Da Ros, M. Pu, et al., "Single-source chip-based frequency comb enabling extreme parallel data transmission," Nat. Photonics, vol. 1, 2018. https://doi.org/10.1038/s41566018-0205-5.

[3] A. L. Gaeta, M. Lipson, and T. J. Kippenberg, "Photonic-chipbased frequency combs," Nat. Photonics, vol. 13, no. 3, pp. 158169, 2019.

[4] T. Fortier and E. Baumann, "20 years of developments in optical frequency comb technology and applications," Commun. Phys., vol. 2, no. 1, pp. 1-16, 2019.

[5] V. Torres-Company, A. Fülöp, M. Mazur, et al., "Laser frequency combs for coherent optical communications," J. Lightwave Technol., vol. 37, no. 7, pp. 1-1, 2019.

[6] M. G. Suh, Q. F. Yang, K. Y. Yang, X. Yi, and K. J. Vahala, "Microresonator soliton dual-comb spectroscopy," Science (80-. ), vol. 354, no. 6312, pp. 600-603, 2016.

[7] M. G. Suh, X. Yi, Y. H. Lai, et al., "Searching for exoplanets using a microresonator astrocomb," Nat. Photonics, vol. 13, no. 1, pp. 25-30, 2019.

[8] D. T. Spencer, T. Drake, T. C. Briles, et al., "An optical-frequency synthesizer using integrated photonics," Nature, vol. 557, no. 7703, pp. 81-85, 2018.

[9] E. Obrzud, M. Rainer, A. Harutyunyan, et al., "A microphotonic astrocomb," Nat. Photonics, vol. 13, no. 1, pp. 31-35, 2019.

[10] Z. L. Newman, Maurice V., T. Drake, et al., "Architecture for the photonic integration of an optical atomic clock," Optica, vol. 6, no. 5, p. 680, 2019.

[11] J. Riemensberger, A. Lukashchuk, M. Karpov, et al., "Massively parallel coherent laser ranging using a soliton microcomb," Nature, vol. 581, no. 7807, pp. 164-170, 2020.

[12] S. A. Diddams, T. Udem, J. C. Bergquist, et al., “An optical clock based on a single trapped $199 \mathrm{Hg}+$ ion," Science (80-. ), vol. 293 , no. 5531, pp. 825-828, 2001.

[13] V. Torres-Company and A. M. Weiner, "Optical frequency comb technology for ultra-broadband radio-frequency photonics," Laser Photonics Rev., vol. 8, no. 3, pp. 368-393, 2014.

[14] P. Trocha, M. Karpov, D. Ganin, et al., "Ultrafast optical ranging using microresonator soliton frequency combs," Science (80-.), vol. 359, no. 6378, pp. 887-891, 2018.

[15] S. A. Diddams, K. Vahala, and T. Udem, "Optical frequency combs: coherently uniting the electromagnetic spectrum," Science (New York, N.Y.), vol. 369, no. 6501, 2020. https://doi. org/10.1126/science.aay3676.

[16] J. Liu, E. Lucas, A. S. Raja, et al., "Photonic microwave generation in the X-and K-band using integrated soliton microcombs," Nat. Photonics, vol. 14, no. 8, pp. 486-491, 2020.

[17] T. Kobayashi, M. Nakamura, F. Hamaoka, et al., “1-Pb/s (32 SDM/46 WDM/768 Gb/s) C-band Dense SDM transmission 
over 205.6-km of single-mode heterogeneous multi-core fiber using 96-Gbaud PDM-16QAM channels," in Optics InfoBase Conference Papers, vol. Part F40-OFC 2017, Los Angeles, Optical Society of America, 2017, p. Th5B.1.

[18] L. Lundberg, M. Mazur, A. Mirani, et al., "Phase-coherent lightwave communications with frequency combs," Nat. Commun., vol. 11, no. 1, pp. 1-7, 2020.

[19] M. Mazur, J. Schröder, M. Karlsson, and P. A. Andrekson, “Joint superchannel digital signal processing for ultimate bandwidth utilization," arXiv:1911.02326v2, 2019.

[20] E. Temprana, E. Myslivets, B. P. Kuo, et al., "Overcoming Kerrinduced capacity limit in optical fiber transmission," Science (80-, vol. 348, no. 6242, pp. 1445-1448, 2015.

[21] L. Galdino, D. Semrau, D. Lavery, et al., "On the limits of digital back-propagation in the presence of transceiver noise," Opt. Express, vol. 25, no. 4, p. 4564, 2017.

[22] D. Semrau, D. Lavery, L. Galdino, R. I. Killey, and P. Bayvel, “The impact of transceiver noise on digital nonlinearity compensation," J. Lightwave Technol., vol. 36, no. 3, pp. 695-702, 2018.

[23] M. P. Yankov, F. Da Ros, E. P. da Silva, et al., "Nonlinear phase noise compensation in experimental WDM systems with 256QAM," J. Lightwave Technol., vol. 35, no. 8, pp. 1438-1443, 2017.

[24] F. Da Ros, M. P. Yankov, E. P. da Silva, et al., "Characterization and optimization of a high-efficiency AlGaAs-on-insulator-based wavelength converter for 64- and 256-QAM signals," J. Lightwave Technol., vol. 35, no. 17, pp. 3750-3757, 2017.

[25] A. Fülöp, M. Mazur, A. Lorences-Riesgo, et al., "High-order coherent communications using mode-locked dark-pulse Kerr combs from microresonators," Nat. Commun., vol. 9, no. 1, p. 1598, 2018.

[26] M. Mazur, M. G. Suh, A. Fülöp, et al., "High spectral efficiency superchannel transmission using a soliton microcomb," in 45th European Conference on Optical Communication (ECOC 2019), Dublin, IET, 2019. Available at: https://doi.org/10.1049/cp. 2019.0883.

[27] G. Rademacher, B. J. Puttnam, R. S. Luís, et al., "10.66 peta-bit/s transmission over a 38-core-three-mode fiber," in 2020 Optical Fiber Communications Conference and Exhibition, OFC 2020 Proceedings, San Diego, Optical Society of America, 2020, p. Th3H.1.

[28] E. U. Rafailov, M. A. Cataluna, and W. Sibbett, "Mode-locked quantum-dot lasers," Nat. Photonics, vol. 1, no. 7, pp. 395-401, 2007.

[29] E. U. Rafailov, M. A. Cataluna, W. Sibbett, et al., “High-power picosecond and femtosecond pulse generation from a twosection mode-locked quantum-dot laser," Appl. Phys. Lett., vol. 87, no. 8, p. 081107, 2005.

[30] G. Liu, Z. Lu, J. Liu, et al., "Passively mode-locked quantum dash laser with an aggregate 5.376 Tbit/s PAM-4 transmission capacity," Opt. Express, vol. 28, no. 4, p. 4587, 2020.

[31] H. A. Haus, “Mode-locking of lasers," IEEE J. Sel. Top. Quant. Electron., vol. 6, no. 6, pp. 1173-1185, 2000.

[32] K. Yvind, D. Larsson, L. J. Christiansen, et al., "Low-jitter and high-power 40-GHz all-active mode-locked lasers," IEEE Photonics Technol. Lett., vol. 16, no. 4, pp. 975-977, 2004.

[33] R. Kaiser and B. Huttl, "Monolithic 40-GHz mode-locked MQW DBR lasers for high-speed optical communication systems," IEEE J. Sel. Top. Quant. Electron., vol. 13, no. 1, pp. 125-135, 2007.
[34] M.-C. Lo, R. Guzmán, and G. Carpintero, "InP femtosecond modelocked laser in a compound feedback cavity with a switchable repetition rate," Opt. Lett., vol. 43, no. 3, p. 507, 2018.

[35] G. Liu, Z. Lu, J. Liu, et al., "A passively mode-locked quantum dot laser with $10.8 \mathrm{tbit} / \mathrm{s}$ transmission over 100-km SSMF," in 2020 Optical Fiber Communications Conference and Exhibition, OFC 2020 - Proceedings, San Diego, Optical Society of America, 2020, p. W2A.2.

[36] J. N. Kemal, P. Marin-Palomo, V. Panapakkam, et al., “WDM transmission using quantum-dash mode-locked laser diodes as multi-wavelength souree and local oscillator," in 2017 Optical Fiber Communications Conference and Exhibition, OFC 2017 Proceedings, Los Angeles, Optical Society of America, vol. 27, no. 22, 2017, pp. 31164-31175.

[37] V. Vujicic, C. Calo, R. Watts, et al., "Quantum dash mode-locked lasers for data centre applications," IEEE J. Sel. Top. Quant. Electron., vol. 21, no. 6, 2015, https://doi.org/10.1109/jstqe. 2015.2487884.

[38] J. N. Kemal, P. Marin-Palomo, K. Merghem, et al., “32QAM WDM transmission using a quantum-dash passively mode-locked laser with resonant feedback," in Optical Fiber Communication Conference Postdeadline Papers, Los Angeles, Optical Society of America, 2017, p. Th5C.3.

[39] P. Marin-Palomo, J. N. Kemal, P. Trocha, et al., "Comb-based WDM transmission at $10 \mathrm{Tbit} / \mathrm{s}$ using a DC-driven quantum-dash mode-locked laser diode," Opt. Express, vol. 27, no. 22, p. 31110, 2019.

[40] Z. Wang, K. Van Gasse, V. Moskalenko, et al., “A III-V-on-Si ultra-dense comb laser," Light Sci. Appl., vol. 6, no. 5, pp. e16260-e16260, 2017.

[41] A. Y. Liu and J. Bowers, "Photonic integration with epitaxial IIIV on silicon," IEEE J. Sel. Top. Quant. Electron., vol. 24, no. 6, 2018.

[42] S. Liu, X. Wu, D. Jung, et al., “High-channel-count $20 \mathrm{GHz}$ passively mode-locked quantum dot laser directly grown on $\mathrm{Si}$ with 4.1 Tbit/s transmission capacity," Optica, vol. 6, no. 2, p. 128, 2019.

[43] D. Hillerkuss, R. Schmogrow, T. Schellinger, et al., "26 Tbit s-1 line-rate super-channel transmission utilizing all-optical fast Fourier transform processing," Nat. Photonics, vol. 5, no. 6, pp. 364-371, 2011.

[44] B. J. Puttnam, R. S. Luís, W. Klaus, et al., “2.15 Pb/s transmission using a 22 core homogeneous single-mode multi-core fiber and wideband optical comb," in 2015 European Conference on Optical Communication (ECOC), Valencia, IEEE, 2015, pp. 1-3.

[45] R. Halir, Y. Okawachi, J. S. Levy, M. A. Foster, M. Lipson, and A. L. Gaeta, "Ultrabroadband supercontinuum generation in a CMOS-compatible platform," Opt. Lett., vol. 37, no. 10, p. 1685, 2012.

[46] B. Kuyken, T. Ideguchi, S. Holzner, et al., “An octave-spanning mid-infrared frequency comb generated in a silicon nanophotonic wire waveguide," Nat. Commun., vol. 6, no. 1, p. 6310, 2015.

[47] M. Yu, B. Desiatov, Y. Okawachi, A. L. Gaeta, and M. Lončar, "Coherent two-octave-spanning supercontinuum generation in lithium-niobate waveguides,” Opt. Lett., vol. 44, no. 5, p. 1222, 2019.

[48] A. R. Johnson, A. S. Mayer, A. Klenner, et al., “Octave-spanning coherent supercontinuum generation in a silicon nitride waveguide," Opt. Lett., vol. 40, no. 21, p. 5117, 2015. 
[49] N. Singh, M. Xin, D. Vermeulen, et al., “Octave-spanning coherent supercontinuum generation in silicon on insulator from $1.06 \mu \mathrm{m}$ to beyond $2.4 \mu \mathrm{m}$," Light Sci. Appl., vol. 7, no. 1 , pp. 17131-17131, 2018.

[50] H. Guo, C. Herkommer, A. Billat, et al., "Mid-infrared frequency comb via coherent dispersive wave generation in silicon nitride nanophotonic waveguides," Nat. Photonics, vol. 12, no. 6, pp. 330-335, 2018.

[51] Y. Okawachi, M. Yu, B. Desiatov, et al., "Chip-based selfreferencing using integrated lithium niobate waveguides," Optica, vol. 7, no. 6, p. 702, 2020.

[52] M. Pu, L. Ottaviano, E. Semenova, and K. Yvind, "Efficient frequency comb generation in AlGaAs-on-insulator," Optica, vol. 3, no. 8, p. 823, 2016.

[53] L. Chang, W. Xie, H. Shu, et al., "Ultra-efficient frequency comb generation in AlGaAs-on-insulator microresonators," Nat. Commun., vol. 11, no. 1, pp. 1-8, 2020.

[54] M. Pu, H. Hu, L. Ottaviano, et al., "Ultra-Efficient and broadband nonlinear AlGaAs-on-insulator chip for low-power optical signal processing," Laser Photonics Rev., vol. 12, no. 12, p. 1800111, 2018.

[55] D. Kong, M. Pu, Y. Liu, et al., "744-nm wavelength conversion of PAM-4 signal using an AIGaAsOI nanowaveguide," Opt. Lett., vol. 45, no. 4, p. 889, 2020.

[56] Z. Tong, A. O. J. Wiberg, E. Myslivets, B. P. P. Kuo, N. Alic, and $S$. Radic, "Spectral linewidth preservation in parametric frequency combs seeded by dual pumps," Opt. Express, vol. 20, no. 16, p. 17610, 2012.

[57] T. J. Kippenberg, S. M. Spillane, and K. J. Vahala, “Kerrnonlinearity optical parametric oscillation in an ultrahigh-Q toroid microcavity," Phys. Rev. Lett., vol. 93, no. 8, p. 083904 , 2004.

[58] T. J. Kippenberg, R. Holzwarth, and S. A. Diddams, "Microresonator-based optical frequency combs," Science, vol. 332, no. 6029, pp. 555-559, 2011.

[59] A. Pasquazi, M. Peccianti, L. Razzari, et al., "Micro-combs: A novel generation of optical sources,” Phys. Rep., vol. 729, pp. 1-81, 2018.

[60] W. Wang, L. Wang, and W. Zhang, "Advances in soliton microcomb generation," Adv. Photonics, vol. 2, no. 03, p. 1, 2020.

[61] H. Bao, A. Cooper, M. Rowley, et al., "Laser cavity-soliton microcombs," Nat. Photonics, vol. 13, no. 6, pp. 384-389, 2019.

[62] A. S. Raja, A. S. Voloshin, H. Guo, et al., "Electrically pumped photonic integrated soliton microcomb," Nat. Commun., vol. 10, no. 1, pp. 1-8, 2019.

[63] G. Lin, A. Coillet, and Y. K. Chembo, "Nonlinear photonics with high-Q whispering-gallery-mode resonators," Adv. Opt. Photonics, vol. 9, no. 4, p. 828, 2017.

[64] D. C. Cole, E. S. Lamb, P. Del'Haye, S. A. Diddams, and S. B. Papp, "Soliton crystals in Kerr resonators," Nat. Photonics, vol. 11, no. 10, pp. 671-676, 2017.

[65] C. Bao, J. A. Jaramillo-Villegas, Y. Xuan, D. E. Leaird, M. Qi, and A. M. Weiner, "Observation of fermi-pasta-ulam recurrence induced by breather solitons in an optical microresonator," Phys. Rev. Lett., vol. 117, no. 16, p. 163901, 2016.

[66] M. Yu, J. K. Jang, Y. Okawachi, et al., "Breather soliton dynamics in microresonators," Nat. Commun., vol. 8, no. 1, pp. 1-7, 2017.

[67] E. Lucas, M. Karpov, H. Guo, M. L. Gorodetsky, and T. J. Kippenberg, "Breathing dissipative solitons in optical microresonators,” Nat. Commun., vol. 8, no. 1, pp. 1-11, 2017.
[68] L. A. Lugiato and R. Lefever, "Spatial dissipative structures in passive optical systems," Phys. Rev. Lett., vol. 58, no. 21, pp. 2209-2211, 1987.

[69] A. B. Matsko, A. A. Savchenkov, W. Liang, V. S. Ilchenko, D. Seidel, and L. Maleki, "Mode-locked Kerr frequency combs," Opt. Lett., vol. 36, no. 15, p. 2845, 2011.

[70] Y. K. Chembo and C. R. Menyuk, "Spatiotemporal Lugiato-Lefever formalism for Kerr-comb generation in whispering-gallery-mode resonators," Phys. Rev. A - At. Mol. Opt. Phys., vol. 87, no. 5, 2013, Art no. 053852.

[71] S. Coen, H. G. Randle, T. Sylvestre, and M. Erkintalo, "Modeling of octave-spanning Kerr frequency combs using a generalized mean-field Lugiato-Lefever model," Opt. Lett., vol. 38, no. 1, p. 37, 2013.

[72] J. S. Levy, A. Gondarenko, M. A. Foster, A. C. Turner-Foster, A. L. Gaeta, and M. Lipson, "CMOS-compatible multiplewavelength oscillator for on-chip optical interconnects," Nat. Photonics, vol. 4, no. 1, pp. 37-40, 2010.

[73] D. J. Moss, R. Morandotti, A. L. Gaeta, and M. Lipson, "New CMOS-compatible platforms based on silicon nitride and Hydex for nonlinear optics," Nat. Photonics, vol. 7, no. 8, pp. 597-607, 2013.

[74] Y. Xuan, Y. Liu, L. T. Varghese, et al., "High-Q silicon nitride microresonators exhibiting low-power frequency comb initiation," Optica, vol. 3, no. 11, p. 1171, 2016.

[75] Y. Okawachi, K. Saha, J. S. Levy, Y. H. Wen, M. Lipson, and A. L. Gaeta, "Octave-spanning frequency comb generation in a silicon nitride chip," Opt. Lett., vol. 36, no. 17, p. 3398, 2011.

[76] M. H. P. Pfeiffer, C. Herkommer, J. Liu, et al., "Octave-spanning dissipative Kerr soliton frequency combs in Si_3N_4 microresonators," Optica, vol. 4, no. 7, p. 684, 2017.

[77] J. Liu, A. S. Raja, M. Karpov, et al., "Ultralow-power chip-based soliton microcombs for photonic integration," Optica, vol. 5, pp. 1347-1353, 2018.

[78] K. Luke, Y. Okawachi, M. R. E. Lamont, A. L. Gaeta, and M. Lipson, "Broadband mid-infrared frequency comb generation in a $\mathrm{Si}_{3} \mathrm{~N}_{4}$ microresonator," Opt. Lett., vol. 40, pp. 4823-4826, 2015.

[79] L. Razzari, D. Duchesne, M. Ferrera, et al., "CMOS-compatible integrated optical hyper-parametric oscillator," Nat. Photonics, vol. 4, no. 1, pp. 41-45, 2010.

[80] X. Yi, Q.-F. Yang, K. Y. Yang, M.-G. Suh, and K. Vahala, "Soliton frequency comb at microwave rates in a high-Q silica microresonator," Optica, vol. 2, no. 12, p. 1078, 2015.

[81] M.-G. Suh and K. Vahala, "Gigahertz-repetition-rate soliton microcombs," Optica, vol. 5, no. 1, p. 65, 2018.

[82] T. Hansson, D. Modotto, and S. Wabnitz, "Mid-infrared soliton and Raman frequency comb generation in silicon microrings," Opt. Lett., vol. 39, no. 23, p. 6747, 2014.

[83] M. Yu, Y. Okawachi, A. G. Griffith, M. Lipson, and A. L. Gaeta, "Mode-locked mid-infrared frequency combs in a silicon microresonator," Optica, vol. 3, no. 8, p. 854, 2016.

[84] B. J. M. Hausmann, I. Bulu, V. Venkataraman, P. Deotare, and M. Loncar, "Diamond nonlinear photonics," Nat. Photonics, vol. 8, no. 5, pp. 369-374, 2014.

[85] H. Jung, R. Stoll, X. Guo, D. Fischer, and H. X. Tang, “Green, red, and IR frequency comb line generation from single IR pump in AIN microring resonator," Optica, vol. 1, no. 6, p. 396, 2014.

[86] Z. Gong, A. Bruch, M. Shen, et al., "High-fidelity cavity soliton generation in crystalline AIN micro-ring resonators," Opt. Lett., vol. 43, no. 18, p. 4366, 2018. 
[87] D. J. Wilson, K. Schneider, S. Hönl, et al., "Integrated gallium phosphide nonlinear photonics," Nat. Photonics, vol. 14, no. 1, pp. 57-62, 2020.

[88] Y. He, Q. F. Yang, J. Ling, et al., "Self-starting bi-chromatic LiNbO 3 soliton microcomb," Optica, vol. 6, no. 9, p. 1138, 2019.

[89] C. Wang, M. Zhang, M. Yu, R. Zhu, H. Hu, and M. Loncar, "Monolithic lithium niobate photonic circuits for Kerr frequency comb generation and modulation," Nat. Commun., vol. 10, no.1, p. $978,2019$.

[90] J. Pfeifle, V. Brasch, M. Lauermann, et al., "Coherent terabit communications with microresonator Kerr frequency combs," Nat. Photonics, vol. 8, no. 5, pp. 375-380, 2014.

[91] P. Marin-Palomo, J. N. Kemal, M. Karpov, et al., "Microresonator-based solitons for massively parallel coherent optical communications," Nature, vol. 546, no. 7657, pp. 274279, 2017.

[92] B. Corcoran, M. Tan, X. Xu, et al., "Ultra-dense optical data transmission over standard fibre with a single chip source," Nat. Commun., vol. 11, no. 1, p. 2568, 2020.

[93] V. Torres-Company, L. K. O. D. Kong, A. A. Jørgensen, et al., "Dark-pulse Kerr ring resonator supporting $1.84 \mathrm{pbit} / \mathrm{s}$ transmission over 37-core fiber," in CLEO 2020, Washington, Optical Society of America, 2020, p. JTh4A.7. Available at: https://doi.org/10.1364/CLEO_AT.2020.JTh4A.7.

[94] E. Obrzud, S. Lecomte, and T. Herr, "Temporal solitons in microresonators driven by optical pulses," Nat. Photonics, vol. 11, no. 9, pp. 600-607, 2017.

[95] H. A. Haus and W. S. Wong, "Solitons in optical communications," Rev. Mod. Phys., vol. 68, no. 2, pp. 423-444, 1996.

[96] L. F. Mollenauer, R. H. Stolen, and J. P. Gordon, "Experimental observation of picosecond pulse narrowing and solitons in optical fibers," Phys. Rev. Lett., vol. 45, no. 13, pp. 1095-1098, 1980.

[97] T. Herr, V. Brasch, J. D. Jost, et al., "Temporal solitons in optical microresonators," Nat. Photonics, vol. 8, no. 2, pp. 145-152, 2014.

[98] V. Brasch, M. Geiselmann, T. Herr, et al., "Photonic chip-based optical frequency comb using soliton Cherenkov radiation," Science (80-. ), vol. 351, no. 6271, pp. 357-360, 2016.

[99] T. J. Kippenberg, A. L. Gaeta, M. Lipson, and M. L. Gorodetsky, "Dissipative Kerr solitons in optical microresonators," Science, vol. 361, no. 6402, 2018. https://doi.org/10.1126/science. aan8083.

[100] B. Stern, X. Ji, Y. Okawachi, A. L. Gaeta, and M. Lipson, "Batteryoperated integrated frequency comb generator," Nature, vol. 562, no. 7727, pp. 401-405, 2018.

[101] A. A. Abidi, "CMOS microwave and millimeter-wave ICs: the historical background," in RFIT 2014 - 2014 IEEE International
Symposium on Radio-Frequency Integration Technology: Silicon Technology Heats Up for THz, Hefei, IEEE, 2014, pp. 1-5. Available at: https://doi.org/10.1109/RFIT.2014.6933267.

[102] B. Shen, L. Chang, J. Liu, et al., "Integrated turnkey soliton microcombs," Nature, vol. 582, no. 7812, pp. 365-369, 2020.

[103] X. Xue, P.-H. Wang, Y. Xuan, M. Qi, and A. M. Weiner, "Microresonator Kerr frequency combs with high conversion efficiency," Laser Photonics Rev., vol. 11, no. 1, p. 1600276 , 2017.

[104] X. Xue, Y. Xuan, Y. Liu, et al., "Mode-locked dark pulse Kerr combs in normal-dispersion microresonators," Nat. Photonics, vol. 9, no. 9, pp. 594-600, 2015.

[105] Y. Liu, Y. Xuan, X. Xue, et al., "Investigation of mode coupling in normal-dispersion silicon nitride microresonators for Kerr frequency comb generation," Optica, vol. 1, no. 3, p. 137, 2014.

[106] M. Karpov, M. H. P. Pfeiffer, H. Guo, W. Weng, J. Liu, and T. J. Kippenberg, "Dynamics of soliton crystals in optical microresonators," Nat. Phys., vol. 15, no. 10, pp. 1071-1077, 2019.

[107] M. Kourogi, N. Ken'ichi, and M. Ohtsu, "Wide-span optical frequency comb generator for accurate optical frequency difference measurement," IEEE J. Quant. Electron., vol. 29, no. 10, pp. 2693-2701, 1993.

[108] K. Beha, D. C. Cole, P. Del'Haye, A. Coillet, S. A. Diddams, and S. B. Papp, “Electronic synthesis of light," Optica, vol. 4, no. 4, p. 406, 2017.

[109] H. Hu, H. C. H. Mulvad, C. Peucheret, et al., " 10 GHz Pulse Source for $640 \mathrm{Gbit} / \mathrm{s}$ OTDM based on $\mathrm{LiNbO}_{3}$ modulators and selfphase modulation," Opt. Express, vol. 19, pp. B343-B349, 2011.

[110] D. R. Carlson, D. D. Hickstein, W. Zhang, et al., "Ultrafast electro-optic light with subcycle control," Science (80-. ), vol. 361, no. 6409, pp. 1358-1363, 2018.

[111] A. Parriaux, K. Hammani, and G. Millot, "Electro-optic frequency combs," Adv. Opt. Photonics, vol. 12, no. 1, p. 223, 2020.

[112] M. Zhang, B. Buscaino, C. Wang, et al., "Broadband electrooptic frequency comb generation in a lithium niobate microring resonator," Nature, vol. 1, 2019. https://doi.org/10.1038/ s41586-019-1008-7.

[113] A. Rueda, F. Sedlmeir, M. Kumari, G. Leuchs, and H. G. L. Schwefel, "Resonant electro-optic frequency comb," Nature, vol. 568, no. 7752, pp. 378-381, 2019.

[114] M. Kourogi, M. Ohtsu, B. Widiyatomoko, and Y. Takeuchi, “Limit of optical-frequency comb generation due to material dispersion," IEEE J. Quant. Electron., vol. 31, no. 12, pp. 2120-2126, 1995. 